\title{
Structural, Metabolic, and Functional Brain Abnormalities as a Result of Prenatal Exposure to Drugs of Abuse: Evidence from Neuroimaging
}

\author{
Florence Roussotte $\cdot$ Lindsay Soderberg • \\ Elizabeth Sowell
}

Received: 9 August 2010 /Accepted: 6 October 2010 /Published online: 28 October 2010

(C) The Author(s) 2010. This article is published with open access at Springerlink.com

\begin{abstract}
Prenatal exposure to alcohol and stimulants negatively affects the developing trajectory of the central nervous system in many ways. Recent advances in neuroimaging methods have allowed researchers to study the structural, metabolic, and functional abnormalities resulting from prenatal exposure to drugs of abuse in living human subjects. Here we review the neuroimaging literature of prenatal exposure to alcohol, cocaine, and methamphetamine. Neuroimaging studies of prenatal alcohol exposure have reported differences in the structure and metabolism of many brain systems, including in frontal, parietal, and temporal regions, in the cerebellum and basal ganglia, as well as in the white matter tracts that connect these brain regions. Functional imaging studies have identified significant differences in brain activation related to various cognitive domains as a result of prenatal alcohol exposure. The published literature of prenatal exposure to cocaine and methamphetamine is much smaller, but evidence is beginning to emerge suggesting that exposure to stimulant drugs in utero may be particularly toxic to dopamine-rich basal
\end{abstract}

\author{
F. Roussotte $\cdot$ L. Soderberg $\cdot$ E. Sowell \\ Developmental Cognitive Neuroimaging Group, \\ Department of Neurology, University of California, \\ Los Angeles, CA, USA \\ F. Roussotte $\cdot$ E. Sowell \\ Interdepartmental Ph.D. Program for Neuroscience, \\ University of California, \\ Los Angeles, CA, USA \\ E. Sowell $(\square)$ \\ Developmental Cognitive Neuroimaging Group, Laboratory of \\ Neuro Imaging, University of California, Los Angeles, \\ 710 Westwood Plaza, Room 1-138, \\ Los Angeles, CA 90095-7332, USA \\ e-mail: esowell@ucla.edu
}

ganglia regions. Although the interpretation of such findings is somewhat limited by the problem of polysubstance abuse and by the difficulty of obtaining precise exposure histories in retrospective studies, such investigations provide important insights into the effects of drugs of abuse on the structure, function, and metabolism of the developing human brain. These insights may ultimately help clinicians develop better diagnostic tools and devise appropriate therapeutic interventions to improve the condition of children with prenatal exposure to drugs of abuse.

Keywords Neuroimaging $\cdot$ Prenatal exposure $\cdot$ Drugs of abuse - Alcohol - Fetal alcohol spectrum disorders . Cocaine $\cdot$ Methamphetamine $\cdot$ Teratogens $\cdot$ Brain development

\section{Introduction}

Prenatal exposure to drugs of abuse, such as alcohol and stimulants, leads to complex alterations in developing trajectory of the central nervous system. Until recently, researchers relied on animal models to study the effects of drugs of abuse on brain development. In recent years, however, important advances in neuroimaging, particularly in non-invasive Magnetic Resonance (MR)-based imaging techniques, have led to a much better understanding of the way in which prenatal exposure to drugs of abuse impact the structure, function, and metabolism of the developing human brain. Magnetic Resonance Imaging (MRI) is the most commonly used brain imaging modality in human scientific research. We will describe recent MR neuroimaging studies examining alterations in the structure, function, or metabolism of the brains of children, adoles- 
cents, and young adults who were prenatally exposed to drugs of abuse; including alcohol, cocaine, methamphetamine, or a combination of these drugs. For each of these drugs, we will briefly describe the pharmacological mechanisms of action, the current theories about the mechanisms underlying teratogenic effects, and summarize the most significant findings from animal models of prenatal exposure, before reviewing the current human neuroimaging findings. Following is a description of each MR-based imaging technique which has been used as a vehicle for understanding the impact of prenatal drug exposure on the developing brain, and which comprises the literature we will discuss in the following sections.

\section{Structural Magnetic Resonance Imaging (sMRI)}

sMRI allows for high-spatial resolution in-vivo measurement of brain structure. In these studies, research participants are exposed to a high intensity magnet, which detects differences between different types of body tissue and produces data that can be reconstructed into visual images. Physical changes specific to different tissue types result in contrast between gray and white matter (Hendee and Morgan 1984). These images can later be analyzed quantitatively using computational techniques and specialized software packages. Measures such as regional volume, cortical thickness, and other measures of brain morphology can be analyzed and compared across groups.

\section{Diffusion Tensor Imaging (DTI)}

DTI also uses MRI technology, and allows measurement of the diffusion of water molecules within brain tissues, specifically white matter, in order to produce images of white matter tracts (Basser et al. 1994). Important variables derived from DTI datasets are Fractional Anisotropy (FA), Mean Diffusivity (MD), and Apparent Diffusion Coefficient (ADC). FA is a weighted measure of water diffusion, and it is believed to relate to the integrity of white matter, with higher values usually indicating increased integrity (i.e., increased myelination, increased axonal density or diameter). It is important to note that, although FA is often thought of as an indirect indication of white matter integrity, FA actually measures spatial coherence of diffusion, which can be affected by other factors besides changes in white matter integrity. Therefore, the interpretation of findings from DTI studies is not always straightforward. MD and ADC are measures of total diffusion. In some cases, higher MD suggests poorer integrity (i.e., less myelination, lower density or disorganization of axonal fibers). Despite limitations, DTI provides important information about changes occurring in white matter microstructure and microdynamics (Le Bihan 1995), during normal brain development, and in various brain pathologies. The main findings from sMRI and DTI studies of prenatal exposure to alcohol and stimulants are summarized in Table 1.

\section{Magnetic Resonance Spectroscopy (MRS)}

MRS is another MR-based neuroimaging method, which provides information about metabolic functioning in particular regions of interest. MRS capitalizes on different physical properties of chemicals in the body when they are exposed to a high magnetic field. MRS can be used to study the metabolism of various organs. This technique is particularly useful in brain research because measures of concentrations of metabolites important to the production of neurotransmitters can be detected. The application of MRS has been mostly limited to proton spectroscopy, which can resolve only a few molecules abundant in the brain. For example, concentrations of neuronal markers such as $\mathrm{N}$ acetyl-containing compounds (NA) or N-acetyl aspartate (NAA) and glutamate-glutamine (GLX), glial markers such as choline (Cho) and myoinositol (MI), as well as chemicals involved in energy metabolism such as creatine $(\mathrm{Cr})$ or total creatine (tCR), can be measured either in absolute concentration values or as ratios relative to each other (Ross and Bluml 2001).

\section{Functional Magnetic Resonance Imaging (fMRI)}

fMRI is one of the most frequently used techniques to study brain function in humans. This technique provides an indirect assessment of neuronal activity. It measures the dynamic regulation of blood flow in the brain, known as the hemodynamic response, which relates to brain activity. Oxygenated hemoglobin has a different MR signal than deoxygenated hemoglobin. Thus, hemodynamic events lead to transient changes in MR signal, which can be observed when nuclear magnetic resonance images of the brain are acquired in rapid succession. Determining which brain regions show an increase in MR signal intensity provides clues as to which areas are activated during a particular cognitive task. fMRI allows researchers to localize functional brain activation with an accuracy of millimeters and a temporal resolution of seconds (Cohen and Bookheimer 1994). Unlike Blood Oxygen Level-Dependent (BOLD) fMRI described above, Arterial Spin Labeling (ASL) perfusion fMRI investigates patterns of cerebral activation by using magnetically labeled arterial blood water as an endogenous tracer to provide an absolute quantification (millimeters of blood per 100 gram of tissue per minute) of Cerebral Blood Flow (CBF), which is coupled with neuronal activity (Detre et al. 1992). 
Table 1 Summary of main sMRI and DTI findings

\begin{tabular}{|c|c|c|c|c|c|c|}
\hline & Frontal Lobes & Temporal Lobes & $\begin{array}{l}\text { Parietal } \\
\text { Lobes }\end{array}$ & Subcortical Structures & Cerebellum & Corpus Callosum \\
\hline \multicolumn{7}{|l|}{ Alcohol } \\
\hline $\begin{array}{l}\text { Archibald et al. } \\
\text { (2001) }\end{array}$ & $\mathrm{V}$ relatively spared & $\mathrm{V}$ relatively spared & $\begin{array}{l}\downarrow \mathrm{GM}, \\
\downarrow \mathrm{WM}\end{array}$ & $\begin{array}{l}\downarrow \text { V Caudate, HPC } \\
\text { relatively spared }\end{array}$ & & \\
\hline Sowell et al. (2002a) & $\downarrow \mathrm{V}$, ventral parts only & & $\begin{array}{l}\uparrow \mathrm{GM} \\
\text { density }\end{array}$ & & & \\
\hline Sowell et al. (2008a) & $\uparrow \mathrm{CT}$ & $\uparrow \mathrm{CT}$ & $\uparrow \mathrm{CT}$ & & & \\
\hline Sowell et al. (2001a) & & $\uparrow \mathrm{GM}, \downarrow \mathrm{WM}$ & $\uparrow \mathrm{GM}, \downarrow \mathrm{WM}$ & & & \\
\hline Sowell et al. (2002b) & & $\begin{array}{l}\text { Altered GM } \\
\text { asymmetry }\end{array}$ & & & & \\
\hline Mattson et al. (1996) & & & & $\downarrow \mathrm{V} \mathrm{BG}, \mathrm{T}, \mathrm{HT}$ & & \\
\hline Cortese et al. (2006) & & & & $\downarrow$ V Caudate & & \\
\hline Sowell et al. (1996) & & & & & $\downarrow \mathrm{A}$ anterior $\mathrm{VM}$ & \\
\hline O’Hare et al. (2005) & & & & & $\begin{array}{l}\downarrow \mathrm{A} \text { anterior } \mathrm{VM} \\
\text { and DP }\end{array}$ & \\
\hline Riley et al. (1995) & & & & & & $\downarrow \mathrm{A}$ or $\mathrm{AG}$ \\
\hline Swayze et al. (1997) & & & & & & $\mathrm{AG}$ \\
\hline $\begin{array}{l}\text { Bookstein et al. } \\
\text { (2001) }\end{array}$ & & & & & & $\begin{array}{l}\text { High shape } \\
\text { variability }\end{array}$ \\
\hline $\begin{array}{l}\text { Bookstein et al. } \\
\text { (2002) }\end{array}$ & & & & & & $\begin{array}{l}\text { High shape } \\
\text { variability }\end{array}$ \\
\hline Sowell et al. (2001b) & & & & & & $\downarrow \mathrm{A}$ and DP \\
\hline Ma et al. (2005) & & & & & & $\downarrow \mathrm{FA}$ \\
\hline Li et al. (2009) & & & & & & $\downarrow$ FA \\
\hline Fryer et al. (2009) & $\downarrow$ FA & & & & & $\downarrow$ FA \\
\hline Wozniak et al. (2006) & & & & & & $\uparrow \mathrm{MD}$ \\
\hline Sowell et al. (2008b) & & $\downarrow \mathrm{FA}$, right & & & & $\downarrow \mathrm{FA}$ \\
\hline Lebel et al. (2008) & $\uparrow \mathrm{MD}$ & $\downarrow \mathrm{FA}, \uparrow \mathrm{MD}$ & & $\begin{array}{l}\downarrow \text { FA T, } \uparrow \text { FA GP, } \\
\uparrow \text { MD GP, Put, T }\end{array}$ & & $\begin{array}{l}\downarrow \mathrm{FA} \\
\downarrow \mathrm{MD}\end{array}$ \\
\hline \multicolumn{7}{|l|}{ Cocaine } \\
\hline Warner et al. (2006) & $\uparrow \mathrm{D}_{\mathrm{av}}$ & & & & & \\
\hline $\begin{array}{l}\text { Dow-Edwards et al. } \\
\text { (2006) }\end{array}$ & & & $\downarrow \mathrm{GM}$ & & & $\downarrow \mathrm{V}$ \\
\hline Rao et al. (2007) & & & & $\begin{array}{l}\uparrow \text { V Amydala, } \\
\downarrow \text { V Caudate }\end{array}$ & & \\
\hline Avants et al. (2007) & & & & $\downarrow$ V Caudate & & \\
\hline \multicolumn{7}{|l|}{ Methamphetamine } \\
\hline Chang et al. (2004) & & & & $\downarrow$ V GP, Put, HPC & & \\
\hline Sowell et al. (2010) & $\begin{array}{l}\downarrow \text { V IFG } \\
\uparrow \text { VAC }\end{array}$ & $\begin{array}{l}\uparrow \mathrm{V} \text { inferior } \\
\text { and medial }\end{array}$ & $\begin{array}{l}\downarrow \mathrm{V} \text { dorsal } \\
\uparrow \mathrm{V} \text { PC }\end{array}$ & $\downarrow$ V Str, T & & \\
\hline Cloak et al. (2009) & $\downarrow \mathrm{ADC}$ & & $\downarrow \mathrm{ADC}$ & & & \\
\hline
\end{tabular}

$V$ Volume, $A$ area, $C T$ Cortical Thickness, $D P$ Displacement, $A G$ Agenesis, $G M$ Gray Matter, $W M$ White Matter, $A C$ Anterior Cingulate, $P C$ Posterior Cingulate, $B G$ Basal Ganglia, Str striatum, GP Globus Pallidus, Put Putamen, HPC Hippocampus, $T$ Thalamus, $H T$ Hypothalamus, VM vermis, $I F G$ Inferior Frontal Gyrus, $F A$ Fractional Anisotropy, $M D$ Mean Diffusivity, $D_{a v}$ Average Diffusion, $A D C$ Average Diffusion Coefficient

\section{Neuroimaging of Prenatal Exposure to Alcohol}

\section{Mechanism of Action and Toxicity}

Alcohol is a central nervous system depressant, which decreases cortical and subcortical activity through its interactions with membrane receptors. Alcohol primarily acts as an indirect agonist at the $\mathrm{GABA}_{\mathrm{A}}$ receptor (Ticku et al. 1986), but it also acts as an indirect antagonist at the glutamate NMDA receptor (Hoffman et al. 1989). Alcohol also facilitates dopamine transmission in the mesolimbic system (Imperato and Di Chiara 1986). Alcohol crosses the placenta and the blood-brain barrier and can exert teratogenic effects on the developing fetus through various mechanisms, including alcohol-induced hypoxia (Mitchell et al. 1998), increased embryonic oxidative stress (Pollard 2007; Ornoy 
2007), disruption of growth-factor signaling (Feng et al. 2005; Miller 2003). In addition, since GABA receptors are present early in embryogenesis (Schwartz and Meinecke 1992) and are involved in the regulation of many developmental processes, such as neurite growth (Borodinsky et al. 2003), interfering with GABA receptors may disrupt the normal course of neurogenesis.

Animal models have demonstrated that prenatal alcohol exposure results in a variety of structural and metabolic brain abnormalities (Miller and Dow-Edwards 1988). Some examples of neurological dysfunction and developmental disturbances include reductions in spinal and cranial motor neuron production and size (Barrow Heaton et al. 1999); disturbance of neurogenesis and/or gliogenesis in the neocortex (Miller and Robertson 1993), cerebellum (Shetty and Phillips 1992), and hippocampus (Redila et al. 2006); increased neuronal cell death (Olney et al. 2000); abnormal neuronal migration and decreased myelination (Ozer et al. 2000); disruptions in the integrity of plasma membrane NMDA receptors (Honse et al. 2003; Hughes et al. 2001); adverse effects on the endocrine and immune system (Zhang et al. 2005; Wilcoxon et al. 2005); and dysregulation of the hypothamic-pituitary-adrenal (HPA) axis (Glavas et al. 2007), which can lead to increased responsiveness to later stressors and reduced plasticity in the hippocampus (Sliwowska et al. 2010).

The negative effects of prenatal alcohol exposure in humans have been extensively documented since the 1970's. Several researchers in the United States reported growth deficiency and developmental delays in children exposed to alcohol in utero, and the term Fetal Alcohol Syndrome (FAS) was introduced (Jones and Smith 1973; Jones et al. 1973). Since that time, much has been learned about the significant and permanent changes caused by the teratogenic effects of alcohol on the developing fetus. Diagnosis of FAS focuses on four criteria: facial dysmorphology (including short palpebral fissures, a long and flat philtrum, and thin upper vermilion); growth deficiency; evidence of CNS dysfunction; and prenatal exposure to alcohol (Astley and Clarren 2000). However, many alcohol-exposed children do not meet full criteria for a diagnosis of FAS. Nevertheless, these children experience significant and debilitating cognitive and behavioral impairments that impact their daily functioning (Mattson and Riley 1998). Thus, a more inclusive term to describe a wide range of symptom severity has recently emerged. Fetal Alcohol Spectrum Disorders (FASD) encompasses the complete spectrum of effects resulting from prenatal alcohol exposure, with or without facial dysmorphisms. This new "umbrella" term includes a wide range of diagnostic categories, such as FAS, Partial FAS (PFAS), Alcohol Related Neurodevelopmental Disorders (ARND), Fetal Alcohol Effects (FAE), and Alcohol Related Birth Defects (ARBD).
Structural and Metabolic Brain Abnormalities as a Result of Prenatal Alcohol Exposure

In recent years, several neuroimaging studies have confirmed that children with heavy prenatal alcohol exposure show reductions in overall brain size (Archibald et al. 2001; Mattson et al. 1996; Swayze et al. 1997). This finding was first reported in post-mortem studies published in the 1970's (Jones and Smith 1973; Jones et al. 1973; Clarren and Smith 1978). The advent of structural neuroimaging techniques has allowed researchers to study localized brain volume reductions and to make inferences about what brain systems are most affected by prenatal exposure to alcohol. In the next several paragraphs, we will summarize results from various neuroimaging studies of prenatal alcohol exposure. It is important to keep in mind that several of these studies used the same sample of subjects with FASD. In particular, various studies by our group relied on the same data, which was analyzed with diverse techniques in order to study different aspects of brain structure, such as brain shape abnormalities, cortical thickness, gray and white matter volumes, gray matter asymmetry, and cerebellar and callosal morphology.

Evidence of structural abnormalities in the frontal lobe as a result of prenatal alcohol exposure has been reported. Although one study found that frontal lobe volumes were relatively spared compared to other parts of the brain after controlling for overall brain size (Archibald et al. 2001), another study, which examined subregions of the frontal lobes, reported volume reductions in subjects with FASD. Specifically, children with FASD had decreased volumes of the ventral frontal lobes, most prominently in the left hemisphere, while larger dorsal frontal regions appeared somewhat spared (Sowell et al. 2002a) (Fig. 1b). A later study by the same group reported another type of structural brain abnormality in the frontal lobes. Children with FASD were found to have increased cortical thickness compared to unexposed controls in ventral and inferolateral frontal regions in the right hemisphere (Sowell et al. 2008a) (Fig. 2).

The published literature also suggests that prenatal alcohol exposure impacts the temporal lobes. Although temporal lobe volumes appear to be relatively spared compared to other brain regions after controlling for overall brain size in children with FASD (Archibald et al. 2001), subtle structural abnormalities of the temporal lobes have been reported. Specifically, children with FASD were found to have increased gray matter and decreased white matter in the temporal cortex (Sowell et al. 2001a). Another study by the same group showed that subjects with heavy prenatal alcohol exposure had increased cortical thickness in the temporal lobes, which suggests increased gray matter in these regions and corroborates the earlier report (Sowell et 


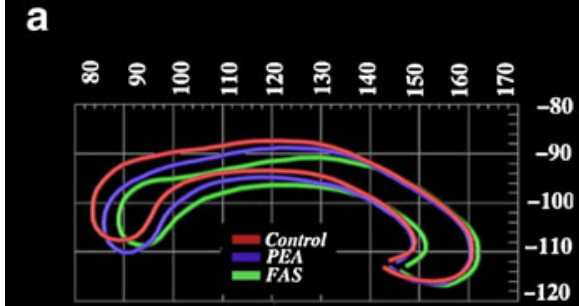

1

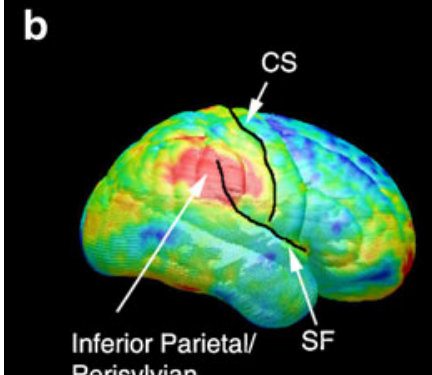

b

Perisylvian

Decreased DFC

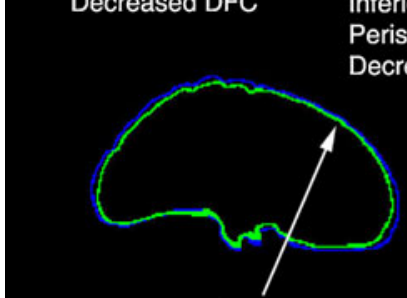

Dorsal Frontal

Lobe Sparing

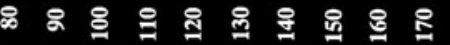

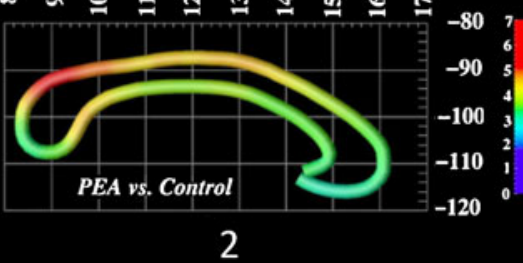

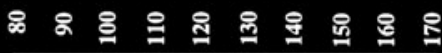

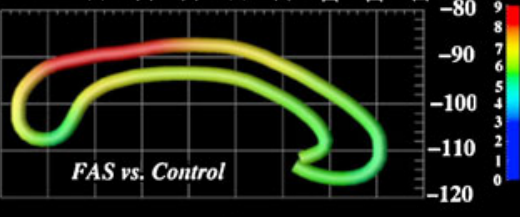

3

\section{6}




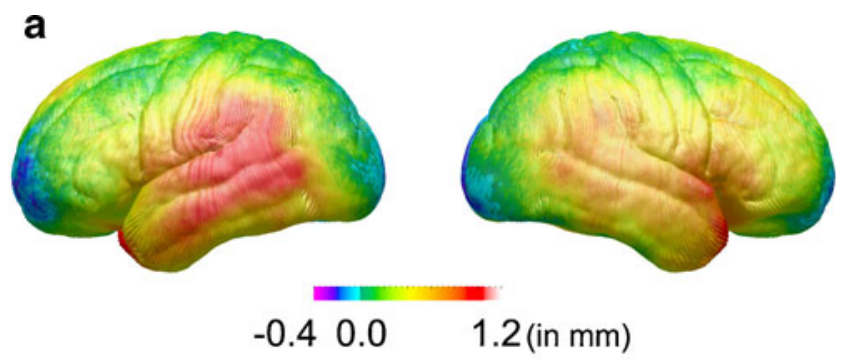

b
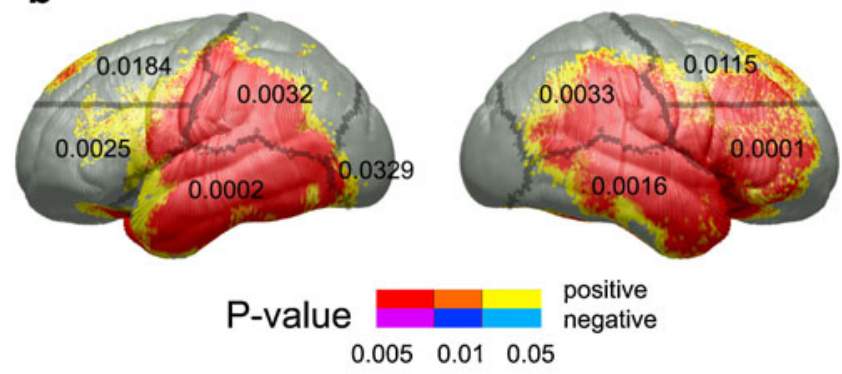

C
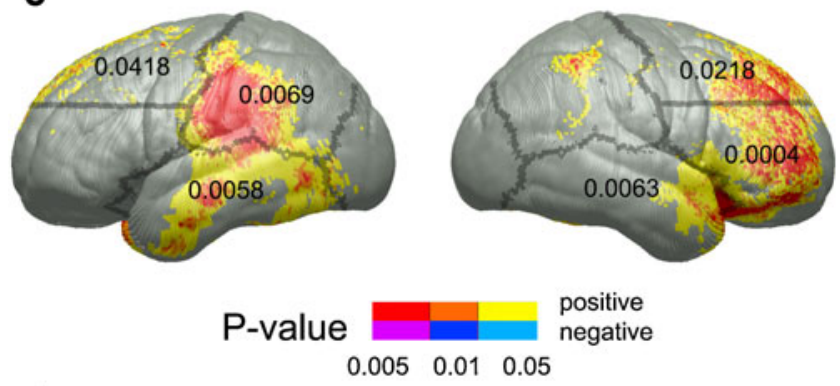

d
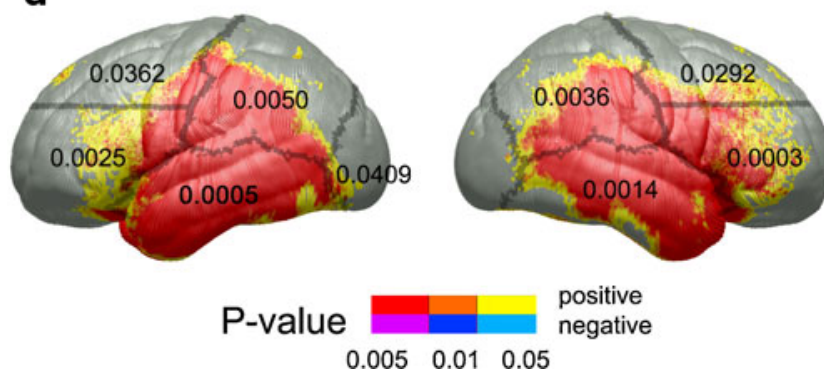

Fig. 2 a FASD versus control maps of the group difference in cortical thickness in millimeters according to the color bar. $\mathbf{b}$ Uncorrected $\mathrm{P}$ maps representing the significance of group differences in cortical thickness for the FASD versus control comparison. Positive correlations are shown in hot colors, and negative correlations are shown in cool colors. There were no regions where the FASD subjects had thinner cortex than the controls. Regions in red are significant at an uncorrected $P$ value of 0.005 or less; in orange, $P$ values range between 0.01 and 0.005 ; and in yellow, $P$ values between range between 0.05 and 0.01. c Uncorrected statistical P maps of the PEA versus control comparison. d Uncorrected statistical P maps of the FAS versus control comparison. The color coding for $\mathbf{c}$ and $\mathbf{d}$ are identical to those described for panel b. The outline of gross ROI boundaries used in permutation analyses are shown superimposed over the left and right lateral surfaces of each of the uncorrected maps, and permutation $P$ values, where significant, are displayed within each ROI (Sowell et al. 2008a) increase in gray matter may result from incomplete synaptic pruning during neural development, but alternatively, it may be a result of unmyelienated white matter, which appears as gray matter on magnetic resonances images (Sowell et al. 2004). Brain development is a very protracted process which continues throughout the lifespan, and the trajectory of maturational effects vary extensively in different regions of the cortex (Sowell et al. 2003) (Fig. 3), with some areas myelinating early, and others continuing myelination into adulthood. In addition, the relationship between cortical thickness and cortical volumes is likely complex, and has not been well characterized in this population, which complicates the interpretation of such findings in individuals with prenatal alcohol exposure even further.

Structural brain abnormalities have also been reported in some subcortical structures in children with prenatal alcohol exposure. The published literature suggests that basal ganglia and diencephalic structures are most affected, while medial temporal lobe regions may be relatively spared. Specifically, decreased volumes of the basal ganglia have been reported, and remain significant, even after controlling for reductions in total brain volume (Mattson et al. 1996). Within the basal ganglia, the caudate appears to be most affected (Archibald et al. 2001; Cortese et al. 2006). Diencephalic structures such as the thalamus and hypothalamus also show volume reductions in children with severe prenatal alcohol exposure (Mattson et al. 1996). Hippocampal volumes, on the other hand, appear to be somewhat preserved relative to overall brain volume reduction in children exposed to alcohol in utero (Archibald et al. 2001).

Two studies have also reported structural changes in the cerebellum associated with prenatal alcohol exposure. An early study reported a significant reduction in the area of the anterior vermis, whereas posterior regions appeared relatively spared (Sowell et al. 1996). Consistent with this finding, a later investigation showed abnormalities in both the size and the location of the vermis in children with FASD. In alcohol-exposed subjects, the anterior vermis and the posterior-inferior lobe showed reduced surface area. There was also a displacement of the anterior vermis, which appeared to be shifted inferiorly and posteriorly in exposed subjects compared to unexposed controls (O'Hare et al. 2005) (Fig. 1c).

Many published studies have found abnormalities in white matter tracts in children with prenatal exposure to alcohol. The corpus callosum alone has been the object of several investigations, and several types of defects have been reported, including partial or complete agenesis and reduced size (Swayze et al. 1997; Riley et al. 1995). It has been suggested that children with FASD, unlike unexposed children, show a high level of variability in the shape of the 


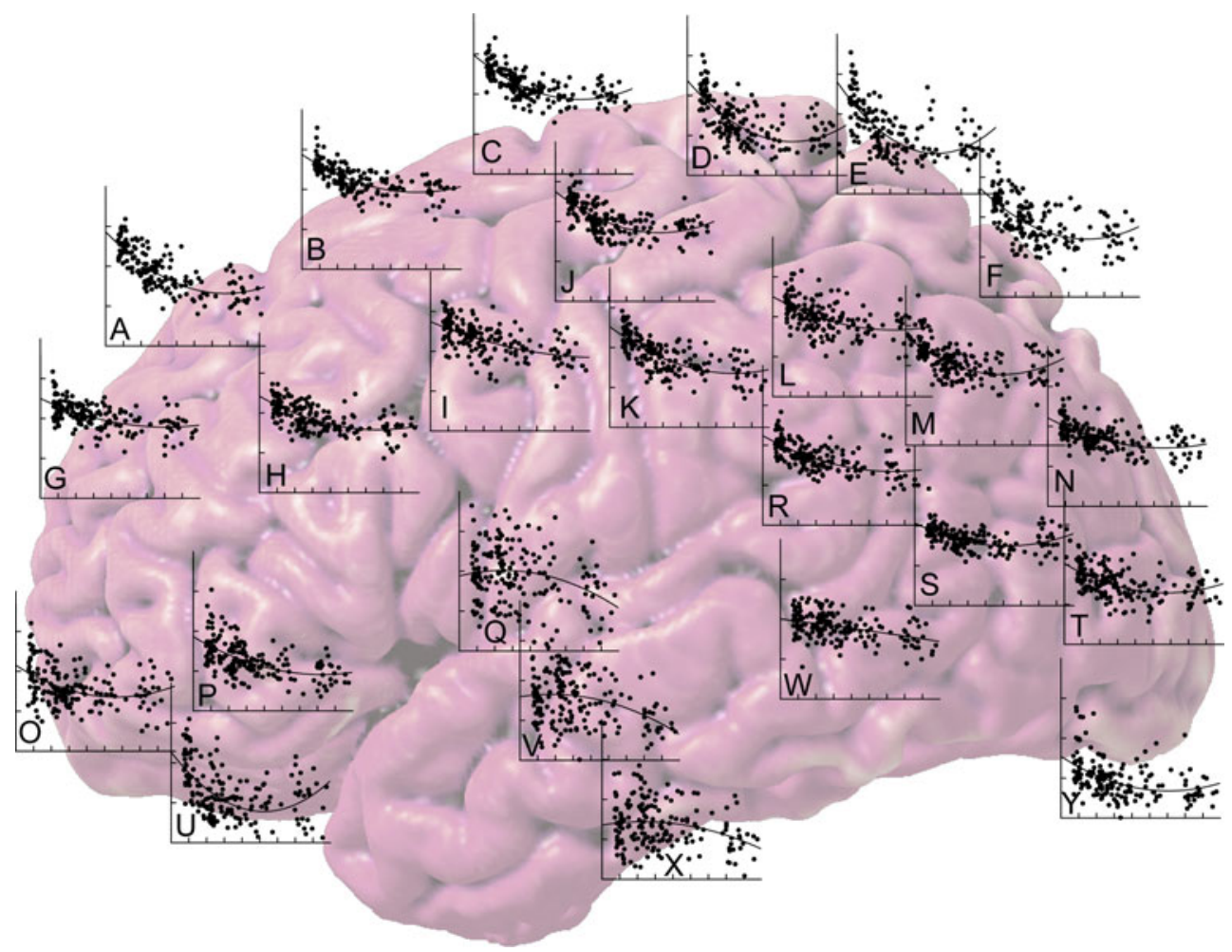

Fig. 3 Shown is a surface rendering of a human brain (left hemisphere; left is anterior, right is posterior) with scatterplots for gray matter density (GMD) at various points over the brain surface where the measurements were taken. Age in years is represented on the $x$-axis (range 0 to 90 ) and GMD on the $y$-axis (range 0.1 to 0.7 ). Anatomical location associated with each graph: $(A)$ superior frontal gyrus, $(B)$ superior frontal sulcus (SFS), middle region, $(C)$ SFS, posterior region, $(D)$ precentral gyrus, $(E)$ postcentral gyrus, $(F)$ superior parietal gyrus, $(G) \mathrm{SFS}$, anterior region, $(H)$ inferior frontal sulcus (IFS), middle region, $(I)$ IFS, posterior region, $(J)$ precentral sulcus, $(K)$ central sulcus, $(L)$ postcentral sulcus, $(M)$ intraparietal sulcus, $(N)$ secondary intermediate sulcus, $(O)$ olfactory gyrus, $(P)$ IFS, anterior region, $(Q)$ Sylvian fissure, $(R)$ primary intermediate sulcus, $(S)$ superior temporal sulcus (STS), ascending branch, $(T)$ transverse occipital sulcus, $(U)$ olfactory sulcus, $(V)$ STS, main body, $(W)$ STS, posterior branch, $(X)$ inferior temporal sulcus, $(Y)$ inferior occipital gyrus (Sowell et al. 2003) corpus callosum (Bookstein et al. 2001), and that this high level of variance has also been found in adults with FASD (Bookstein et al. 2002). A study found structural abnormalities in the location and size of the corpus callosum in children, adolescents, and young adults with FASD. The inferior and anterior displacement of the corpus callosum in exposed subjects was mostly restricted to posterior splenial and isthmus regions, while the more anterior areas of the genu and mid-body appeared relatively spared. There was also a reduction in the area of the corpus callosum in subjects with FASD, mostly in the posterior region encompassing the splenium (Sowell et al. 2001b) (Fig. 1a).

Not only does the corpus callosum show morphological differences in structural MRI studies of FASD, it also reveals reductions in white matter integrity in DTI investigations. Studies have reported lower FA values in the genu and splenium (Ma et al. 2005), as well as in the isthmus ( $\mathrm{Li}$ et al. 2009) of young adults with severe prenatal alcohol exposure, suggesting decreased integrity of white matter fibers in these callosal regions. Lower FA was observed in the body of the corpus callosum as well as in other white matter tracts, located in bilateral medial frontal and occipital regions in children and adolescents exposed to alcohol prenatally (Fryer et al. 2009). Greater MD was reported in the isthmus of children with prenatal alcohol exposure without any facial abnormalities, suggesting that exposure to alcohol in utero may be associated with microstructural abnormalities in the posterior corpus callosum even in the absence of facial dysmorphology (Wozniak et al. 2006).

In addition to the corpus callosum, abnormalities in other white matter tracts have been reported in children and adolescents with prenatal alcohol exposure. One study using a combination of DTI and structural MRI evaluated both FA, which reflects the quality of the organization of white matter microstructures, and white matter density, which is used to assess white matter macrostructure, by examining the location and amount of white matter throughout the brain. Investigators reported that FA was significantly lower in the exposed group compared to the 
unexposed control group in several areas, in particular in the lateral splenium, in the deep association fibers of the right temporal lobe, and in the right internal capsule and brainstem regions. White matter density was lower in subjects with prenatal alcohol exposure in some, but not all regions where FA was affected (Sowell et al. 2008b). Another DTI study reported abnormal diffusion parameters in many areas of the brain in children with FASD. The right cingulum, bilateral inferior and superior longitudinal fasciculus, splenium of the corpus callosum and left thalamus showed FA reduction in exposed children, while increased FA was observed in the bilateral globus pallidus. Increased MD was noted in the bilateral inferior frontooccipital fasciculus, left inferior longitudinal fasciculus, right cortico-spinal tract, globus pallidus, left putamen, and left thalamus, while MD was reduced in the genus of the corpus callosum, suggesting widespread microstructural differences between exposed subjects and unexposed controls (Lebel et al. 2008).

Finally, three MRS studies reported metabolic abnormalities in the brains of children with prenatal exposure to alcohol, but have yielded inconsistent results. One investigation found lower NAA/Cho and NAA/Cr ratios in the parietal and frontal cortices, frontal white matter, corpus callosum, thalamus, and cerebellar dentate nucleus of adolescents and young adults with FASD, compared to unexposed controls. This was due to increased intensity of the glial markers Cho and $\mathrm{Cr}$, while no changes in the neuronal marker NAA were detected, suggesting that these metabolic abnormalities in subjects with FASD represent changes in glial cells rather than in neurons (Fagerlund et al. 2006). Another study, which examined children rather than adolescents, reported somewhat contradictory findings. A higher $\mathrm{NAA} / \mathrm{Cr}$ ratio was observed in the caudate nucleus of exposed subjects, and this change was due to an increase in NAA alone. This suggests a lack of normal programmed cell death, dendritic pruning, or myeliation in subjects with FASD (Cortese et al. 2006), perhaps consistent with the interpretation of cortical thickness increases being associated with a lack of normal synaptic pruning. A third group made observations that differed from both studies described above. Investigators reported reduced concentrations of Cho in fronto-parietal white matter regions lateral to the midsection of the corpus callosum in children with FASD. Decreased Cho concentrations were associated with reduced frontal white matter volumes and shorter length of the corpus callosum (Astley et al. 2009a).

To our knowledge, six studies have examined relationships between structural brain abnormalities and neurocognitive performance in children with prenatal alcohol exposure. One study investigated relationships between cortical thickness and two measures of cognitive function- ing: verbal learning and visuospatial functioning in subjects with FASD and unexposed controls. Subjects with prenatal alcohol exposure performed more poorly than controls on both tasks. In the control group, negative correlations were reported between verbal recall performance and cortical thickness at the superior edge of the motor strip bilaterally in the left occipital lobe. In the FASD group, positive correlations were found between verbal recall scores and bilateral dorsal prefrontal regions. In the control group, negative correlations were observed between visuospatial skills and cortical thickness in bilateral parietal, left occipital, right dorsal and ventral frontal, as well as in temporal cortices. No correlations between visuospatial skills and cortical thickness were found in subjects with FASD. Taken together, these findings suggest that pruning and myelination processes as well as brain-structurefunction relationships follow an altered trajectory in children with heavy prenatal alcohol exposure (Sowell et al. 2008a). Another study examined relationships between the morphology of the cerebellar vermis and verbal learning as well as visuospatial memory scores in subjects with FASD. The superior edge of the anterior vermis showed the strongest negative correlation with verbal learning performance. This region partially overlaps with the vermal region showing the greatest amount of displacement in subjects with FASD. This suggests that impaired verbal learning may be a cognitive correlate of cerebellar vermal displacement (O'Hare et al. 2005).

The remaining correlational studies examined relationships between white matter structures and cognitive performance. One study examined relationships between callosal dysmorphology, verbal learning abilities, and visuospatial skills in children, adolescents, and young adults with FASD. Results revealed that anterior displacement of the corpus callosum was strongly associated with poorer verbal learning performance. Correlations between callosal dysmorphology and visuospatial skills, on the other hand, were much weaker (Sowell et al. 2001b). Another study examined the cognitive correlates of callosal dysmorphology in children with FASD. Investigators assessed the efficiency of interhemispheric transfer of tactile information in children with FASD. Children with prenatal alcohol exposure made significantly more transfer-related errors than controls. Furthermore, there was a negative correlation between the number of transfer-related errors and the size of the isthmus and splenium, suggesting that callosal abnormalities associated with heavy prenatal alcohol exposure impair the functioning of the corpus callosum (Dodge et al. 2009).

Two additional studies investigated relationships between white matter structures and intellectual functioning. One DTI study attempted to relate white matter integrity, visuomotor integration skills, and reading abilities in 
children with FASD and unexposed controls. Correlations between reading scores and FA were not significant in any region tested. On the other hand, poorer performance on a test of visuomotor integration was significantly associated with lower FA values in the lateral splenium and parietal white matter bilaterally, in the exposed group only. These results show that some white matter microstructural abnormalities observed in children with prenatal alcohol exposure may be clinically significant (Sowell et al. 2008b) (Fig. 4). Finally, a recent DTI study examined relationships between white matter structure and mathematical skills in children with FASD. Significant correlations between math scores and FA were observed. Specifically, investigators reported two positively correlated clusters in the left parietal region, one positively-correlated cluster in the left cerebellum, and one negatively-correlated cluster in the bilateral brainstem. These findings provide additional evidence for a link between white matter microstructure and cognitive functioning in children with heavy prenatal alcohol exposure (Lebel et al. 2010) (Fig. 5).

Functional Brain Abnormalities as a Result of Prenatal Alcohol Exposure

Although a large number of studies have been published about structural brain abnormalities as a result of prenatal alcohol exposure, only six studies have investigated abnormalities in functional brain activation in subjects with FASD, four of which have examined functional brain activation during working memory. The first fMRI study, which focused on spatial working memory, reported that both children and adults with FASD showed greater activity than controls in the inferior and middle frontal cortex, suggesting improper functioning of prefrontal areas in the exposed group. Children with FASD also had greater activation than unexposed children in the parietal cortex
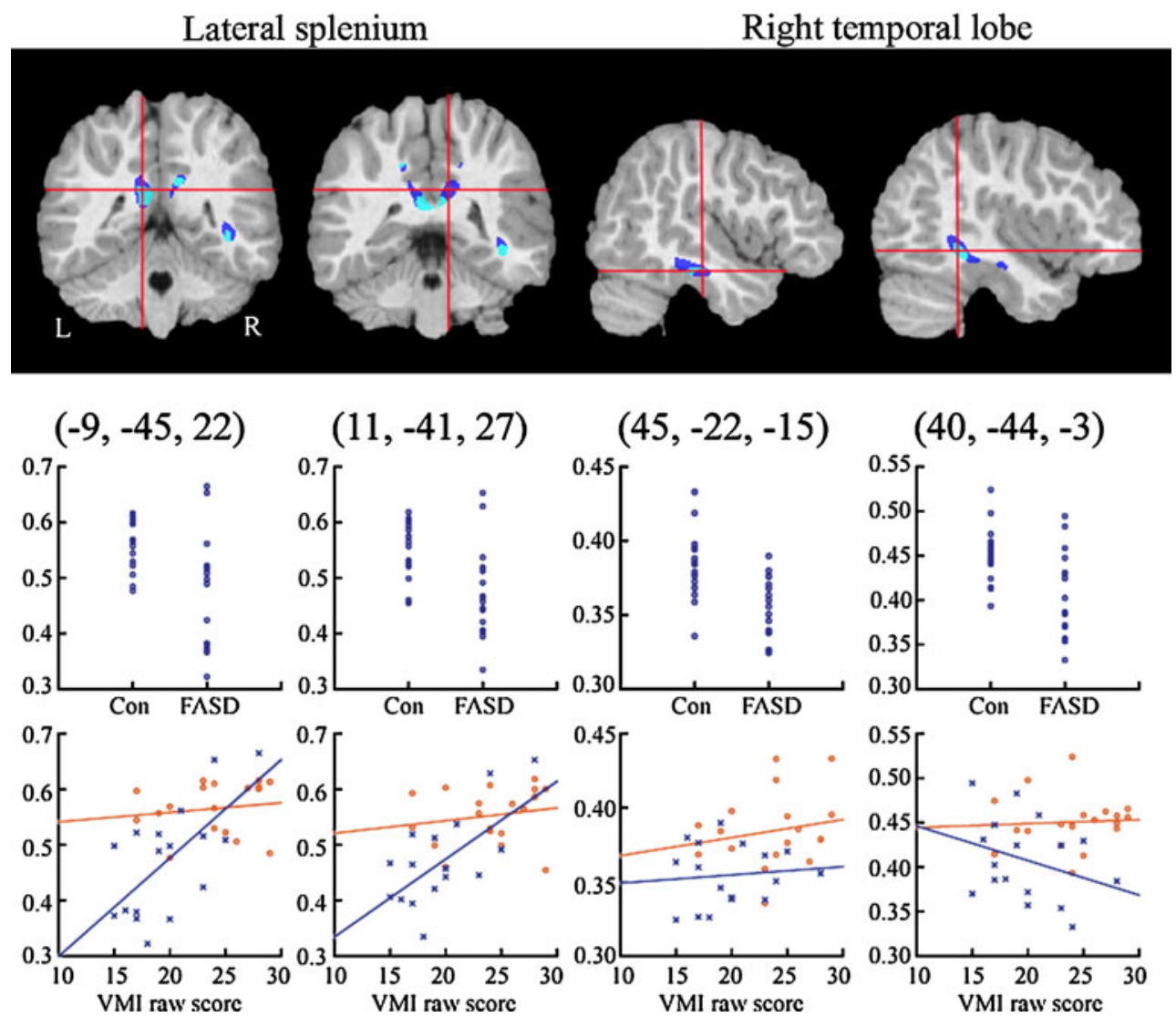

Fig. 4 ROI FA analyses and brain behavior correlations. Coronal and sagittal slices of one representative subject's brain with significant clusters from the FA and white matter density analyses. Shown in dark blue are regions where FA (but not WMD) was significantly lower in the individuals with FASD relative to control subjects $(p<0.05$, FDR correction for multiple comparisons). Regions in light blue are regions where WMD was lower in the FASD relative to controls $(p<0.001$, uncorrected) and overlapped with regions of lower FA in FASD. The yaxes for all graphs have FA values. In the top row of graphs, FA values for each subject are shown for the FASD and control groups. FA values were taken from ROIs (localized approximately at the intersection of the crosshairs in each image) of all voxels within an $8 \mathrm{~mm}$ sphere in the unsmoothed, white matter masked FA volume for each individual. In the bottom row, FA values from the same ROIs are shown in relation to subjects behavioral scores on the VMI. Control subjects are shown in red and FASD subjects in blue. Correlations between VMI scores and FA were highly significant in the left and right lateral splenium ROIs within the FASD, but not control subjects, and not for either group in the anterior and posterior temporal ROIs (Sowell et al. 2008) 


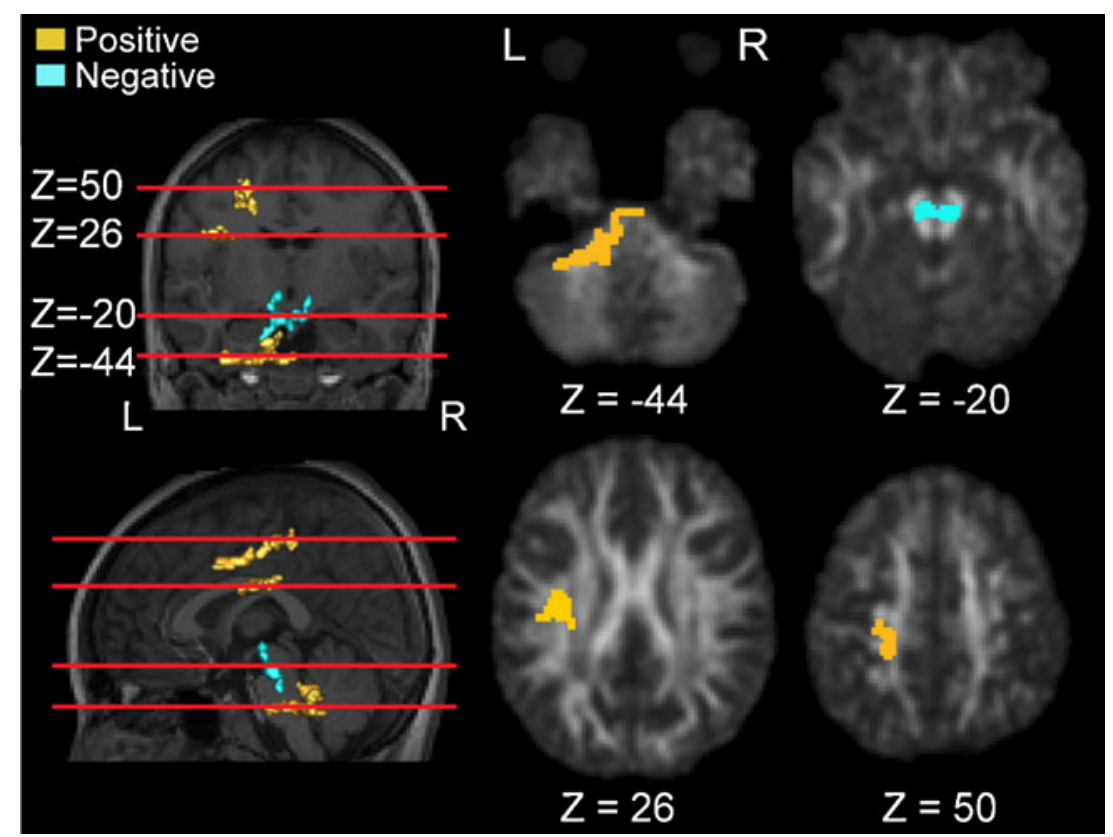

Fig. 5 Significantly correlated clusters of FA versus math ability in 21 fetal alcohol spectrum disorder (FASD) children. The locations of the four significantly correlated clusters are shown in yellow (positive correlations) and cyan (negative correlation). On the left, each cluster is shown with a backdrop of a coronal and a sagittal slice (T1weighted images). On the right, one slice through each cluster is shown, with the significantly correlated voxels in that slice overlaid onto an individual FA map. The location of each axial slice is shown as a red line through the coronal and sagittal slices. MNI coordinates of each slice are given. All images shown are normalized images from a 10-year-old girl with FASD who scored 75 on a Quantitative Concepts test (Lebel et al. 2010) in one of the task conditions (Malisza et al. 2005). However, since accuracy and reaction time were poorer in exposed subjects, it is difficult to determine whether the observed differences in brain activation reflect actual group effects, or just general factors related to performance. Nonetheless, some of these findings have been replicated in a more recent fMRI study of spatial working memory in children and adolescents with FASD. Investigators reported group differences in brain activation despite equivalent performance on the task in children with prenatal alcohol exposure and controls. As in the previous study, they observed greater functional activation in exposed subjects in inferior frontal regions during spatial working memory. They also noted increased activation in other areas, including many subcortical regions, in children with FASD compared to unexposed controls (Spadoni et al. 2009). Taken together, these data suggest that prefrontal regions may be taxed to a greater degree during spatial working memory task in subjects with heavy prenatal alcohol exposure.

Two additional fMRI studies have investigated the effects of prenatal alcohol exposure on brain activation during working memory. Despite equivalent performances between groups, children and adolescents with FASD showed increased activation relative to unexposed controls in left dorsal frontal and left inferior parietal cortices, and in posterior temporal regions bilaterally during a verbal working memory task (O’Hare et al. 2009) (Fig. 6). This study relied on a sample of FASD subjects overlapping with the sample used in the DTI study that examined relationships between callosal dysmorphology, verbal learning abilities, and visuospatial skills (Sowell et al. 2008b). Interestingly, some of the areas where verbal workingmemory-related abnormalities in functional activation were detected in this study, in particular posterior temporal and inferior parietal regions (O'Hare et al. 2009), had previously been reported to show structural abnormalities, specifically increased gray matter in a non-overlapping sample of individuals with FASD (Sowell et al. 2002a). The fourth functional neuroimaging study of prenatal alcohol exposure and functional brain activation during working memory focused on visual working memory. Investigators tried to determine whether fMRI could distinguish between diagnostic subclasses within the FASD spectrum. Results showed that in the harder task condition, the most severely affected children had significantly lower activation than controls in various parts of the working memory network, such as the right posterior parietal lobe, right dorsolateral prefrontal cortex, and right middle frontal regions. As one advanced across the diagnostic categories from most to least severe FASD diagnoses and then to controls, activation levels increased in these brain region (Astley et al. 2009b). 
Fig. 6 Surface renderings and coronal sections displaying group average activation for control participants (a), group average activation for alcohol-exposed participants (b), group differences in activation (c), and the difference between these groups when IQ differences are statistically controlled (d) for WM-related activation. Shown here are regions of significant $[Z>1.7$ and a (corrected) cluster significance threshold of $P=0.05]$ activation collapsed across medium and high working memory loads. Regions shown in panels $\mathbf{c}$ and $\mathbf{d}$ represent regions where alcohol-exposed subjects display greater activation relative to control subjects. Voxels are displayed on the brain surface only if they intersect with the surface. $Y$ values correspond to MNI standard space coordinates (O’Hare et al. 2009)
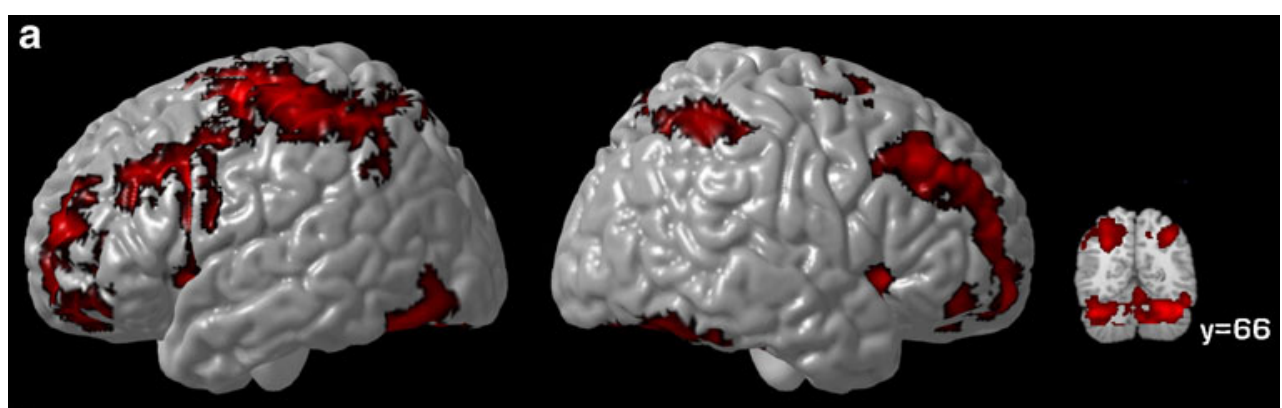

b
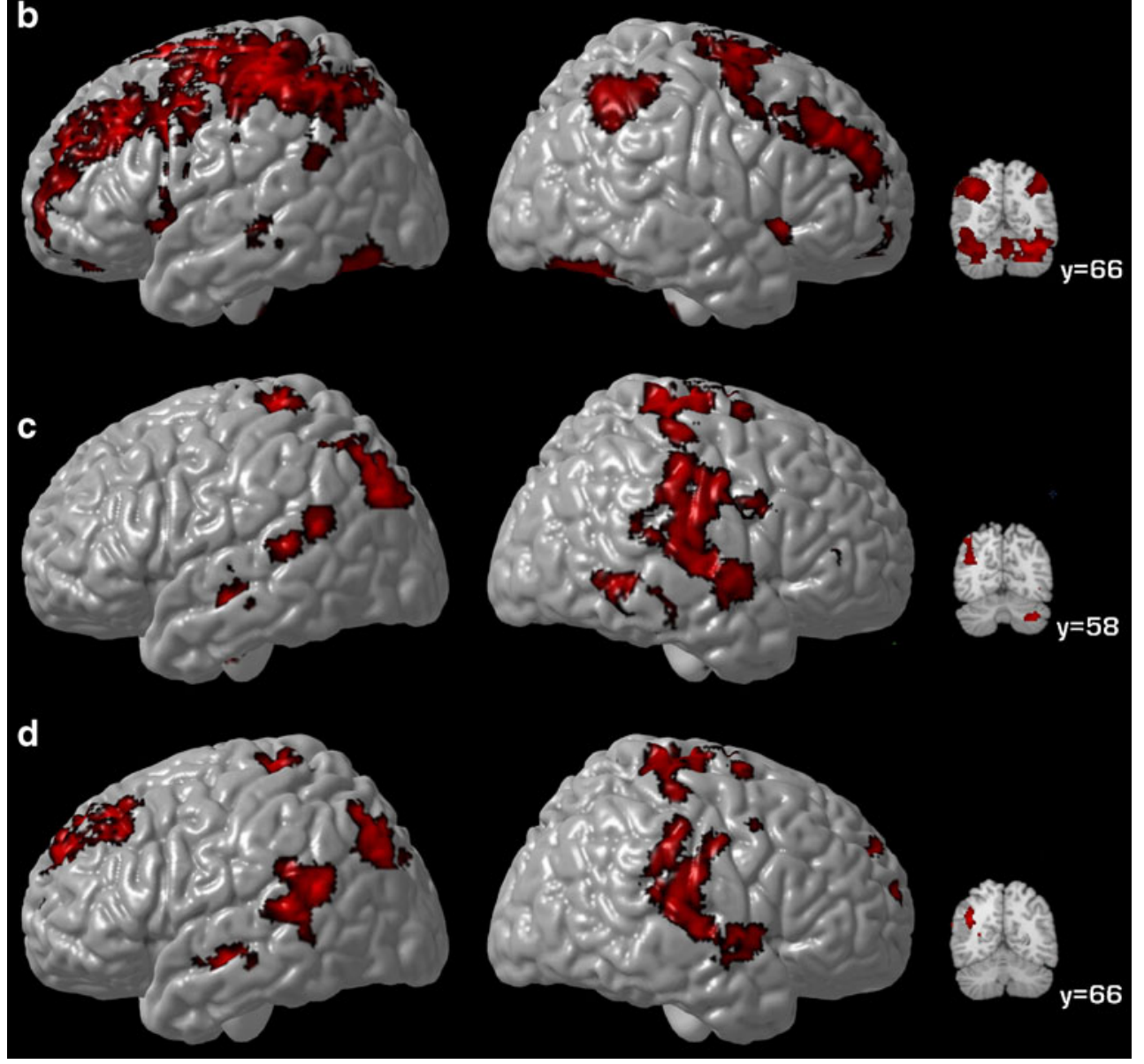

The remaining two fMRI studies examining the effects of prenatal alcohol exposure focused on inhibitory control and verbal learning. Despite the absence of group differences in performance, individuals with FASD showed increased functional activation relative to controls in the prefrontal cortex and decreased activation in the caudate nucleus during a response inhibition task. The authors suggested that this increased recruitment of prefrontal regions might mitigate decreased frontal-striatal network efficiency induced by alcohol teratogenesis (Fryer et al. 2007). On a task of verbal learning, fMRI results indicated that children with prenatal alcohol exposure showed significantly less activation than unexposed controls in the left medial and posterior temporal regions and significantly more activation in the right dorsal frontal cortex, even when group differences in IQ and memory performance were statistically controlled (Sowell et al. 2007) (Fig. 7). In this study, which relied on a sample of FASD subjects overlapping with the samples used in two other studies described above (Sowell et al. 2008b; O'Hare et al. 2009), it appears again that some of the brain regions in which children with FASD showed functional activation abnormalities had previously been shown to present structural abnormalities in a non-overlapping sample of children with heavy prenatal alcohol exposure. In particular, increased gray matter had been reported in medial temporal (Archibald et al. 2001) and posterior temporal regions (Sowell et al. 2002a) in subjects with FASD. 


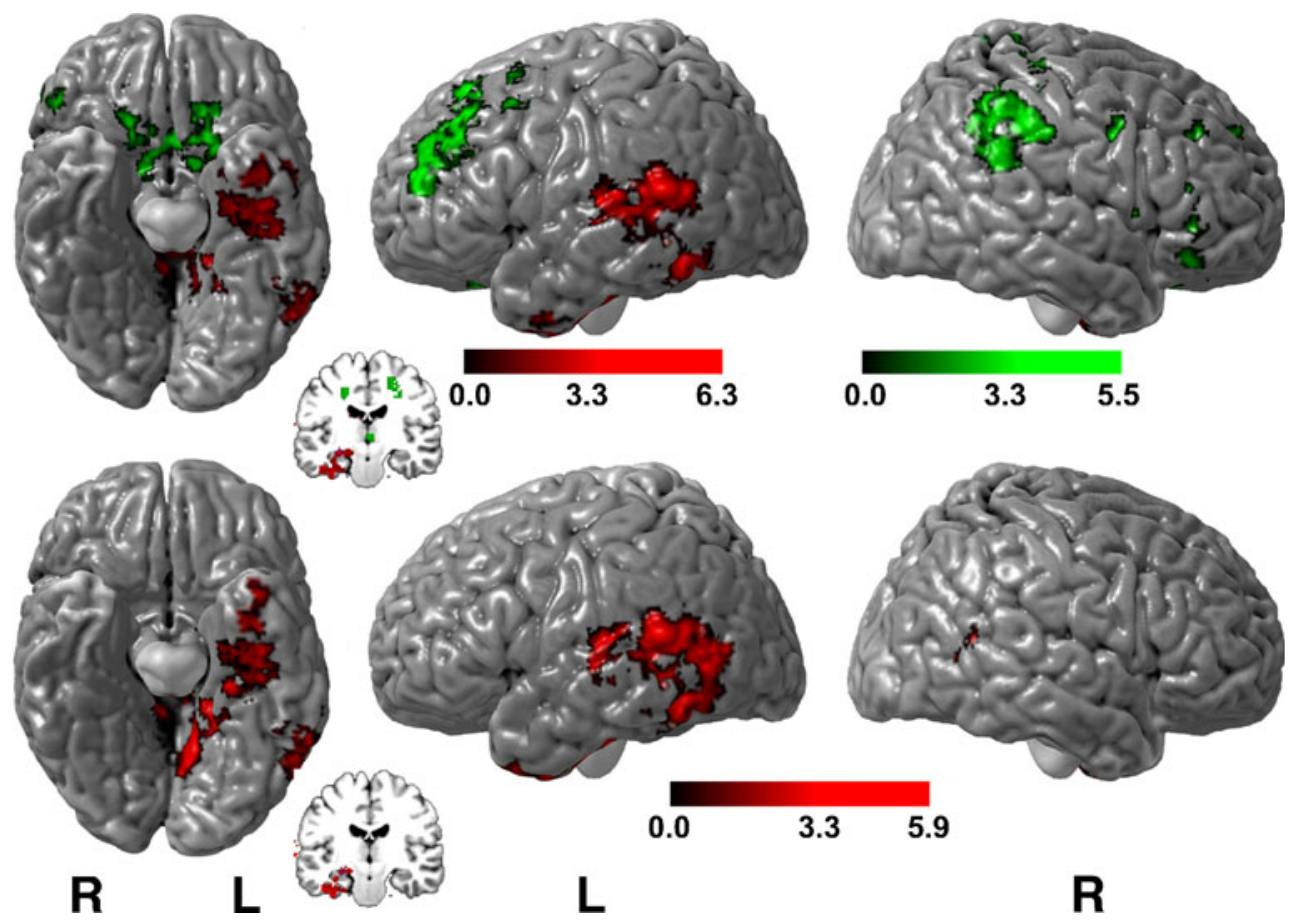

Fig. 7 The top panel illustrates group differences between the alcohol-exposed and the entire control group during verbal learning versus rest. The bottom panel shows group differences between the alcohol-exposed and the 'IQ matched' control group. For all maps, $Z$ values are displayed in color according to the color bars with voxel thresholds of $Z>1.7$ and a (corrected) cluster significance threshold of $P=0.05$. Maps show group differences where $Z$ values are displayed in color according to the color bars with significant alcohol-exposed $>$ control regions shown in green and control $>$ alcohol-exposed regions shown in red. Note that significant voxels were mapped onto the surface only if they intersected with the brain surface, and blurring was not used to map deeper regions of activation onto the surface. Regions of significant activation are also shown mapped onto coronal slices where activation can be seen throughout the brain volume. Note the significant decrease in medial temporal lobe activation in the alcohol-exposed group (shown in red) in maps. Also note the increase in activation in the left frontal lobes in the alcohol-exposed relative to controls (shown in green) (Sowell et al. 2007)
Consistent across all but one of these studies, there appears to be increased activation in frontal brain regions in children with FASDs compared to controls, regardless of fMRI task (Malisza et al. 2005; Spadoni et al. 2009; O'Hare et al. 2009; Fryer et al. 2007; Sowell et al. 2007). It is unclear why increased frontal activation was not reported in one of the fMRI studies (Astley et al. 2009b), as the frontal lobes are an important part of the working memory network. It is possible that differences in methodology between studies may explain this discrepancy. The study with incongruent findings examined regions of interest whereas whole brain analyses are more typically reported in the current literature.

In conclusion, the abundant neuroimaging literature of prenatal alcohol exposure suggests that most brain systems are affected by the teratogenic effects of alcohol. The functional neuroimaging studies published so far indicate that subjects with FASD show abnormal patterns of brain activation in many cognitive domains. These human neuroimaging findings are consistent with the animal literature, which shows that prenatal alcohol exposure affects the developing nervous system through various cellular and molecular mechanisms and results in structural and metabolic abnormalities in many brain regions.

\section{Neuroimaging of Prenatal Exposure to Cocaine}

\section{Mechanism of Action and Toxicity}

Cocaine binds to and blocks the activity of monoamine transporters, including the norepinephrine (NET) and serotonin (SERT) transporters, with highest affinity for the dopamine (DAT) transporter. This results in decreased monoamine re-uptake at the synapse, and increased extracellular levels of these neurotransmitters, especially dopamine (Ritz et al. 1990; Meyer et al. 1993). Cocaine is also a potent anesthetic that blocks sodium channels in a voltage-dependent manner (Wang 1988), resulting in decreased action potentials in excitable cells. In addition, cocaine causes vasoconstriction, which could lead to reduced blood supply and oxygenation to a developing fetus, potentially resulting in chronic or intermittent hypoxia and possible ischemic injury (Koegler et al. 1991; 
Woods et al. 1987). Because cocaine easily crosses both the placenta and the blood-brain barrier (Schenker et al. 1993), all of these mechanisms of action can exert significant teratogenic effects on the developing fetus, both directly and indirectly (Woods 1996). In addition, it has recently been proposed that cocaine may act as an intrauterine stressor, which disrupts the regulation of the neuroendocrine environment by acting on the hypothalamic-pituitary-adrenal (HPA) axis, resulting in altered genetic programming of fetalplacental development (Lester and Padbury 2009).

Various animal studies have suggested that abnormalities related to prenatal exposure to cocaine may result from interference with the neurotrophic functions of monoamine neurotransmitters during brain development (Levitt et al. 1997; Whitaker-Azmitia 1998; Mayes 1999; Malanga and Kosofsky 1999; Stanwood and Levitt 2004). Cocaine may also disrupt early brain development through its interactions with other neurotransmitters, such as GABA (Crandall et al. 2004) and adenosine (Kubrusly and Bhide 2010), and its secondary effects on gene expression, including transcription factor genes (Guerriero et al. 2005; Bhat and Baraban 1993), and apoptosis-related genes (Novikova et al. 2005). The precise mechanisms through which cocaine affects brain development are still debated, but animal models have clearly established that prenatal cocaine exposure results in specific cellular and molecular changes. These include altered cell proliferation (Gressens et al. 1992; Lidow and Song 2001a, b) and migration (Crandall et al. 2004; Lidow 1995; Ren et al. 2004) as well as abnormalities in dopamine D1 receptors and dysfunctions in associated G-protein signaling pathways (Friedman et al. 1996; Jones et al. 2000; Wang et al. 1995; Stanwood et al. 2005).

Structural and Metabolic Brain Abnormalities as a Result of Prenatal Cocaine Exposure

A variety of gross CNS abnormalities have been reported in children with prenatal exposure to cocaine, including cortical infarcts and neural tube defects (Heier et al. 1991), pachygyria (Gomez-Anson and Ramsey 1994), schizencephaly and interhemispheric cysts (Gieron-Korthals et al. 1994), and subependymal and periventricular cysts (Cohen et al. 1994). Regarding more subtle alterations in brain structure, human structural neuroimaging studies of prenatal cocaine exposure have yielded somewhat inconsistent results. A DTI study reported that children with prenatal cocaine exposure had higher average diffusion coefficients in the left frontal callosal and right frontal projection fibers, suggesting suboptimal white matter development due to reduced integrity and/or slower maturation of frontal brain regions (Warner et al. 2006). A study combing sMRI and MRS found no gross structural or volumetric abnormalities in exposed children and adolescents, but reported a $13 \%$ increase in right frontal white matter creatine levels, suggesting abnormal energy metabolism (Smith et al. 2001a).

One whole-brain volumetric study of children with prenatal cocaine exposure showed that exposed subjects had reduced cortical gray matter volumes, total parenchymal volumes, and head circumference. However, these effects lost statistical significance after exposure to other drugs of abuse were regressed out (Rivkin et al. 2008). On the other hand, in another volumetric study, which reported gray matter reductions in occipital and parietal lobes and white matter reductions in the volume of the corpus callosum in exposed children, the findings remained significant even after controlling for exposure to other dugs (Dow-Edwards et al. 2006). In this study, the amount of prenatal cocaine exposure predicted the total area of the corpus callosum, but reductions in gray matter volumes in the occipital and parietal lobe were not dose-dependent (Dow-Edwards et al. 2006). Regarding subcortical structures, a study reported increased gray matter volumes in the amygdala (Rao et al. 2007), and two studies reported decreased volumes in the caudate nucleus (Rao et al. 2007; Avants et al. 2007).

Among the studies published thus far, there have been two reports that changes in brain structures in children with prenatal exposure to cocaine relate to neurocognitive performance. In one study, gray matter volume reductions in the right parietal lobe at 7-8 years were significantly associated with poorer performance on tests of visual attention, visual motor precision, sensorimotor core abilities, and syntax construction at 6 years-of-age. Gray matter volume reductions in the left occipital lobe at 7-8 years were significantly associated with poorer performance on neonatal tests of visual attention, lower scores on visual recognition tests at 12 months, and decreased visuomotor performance at 6 years in children with prenatal cocaine exposure (Dow-Edwards et al. 2006). Another study reported that children exposed to cocaine in utero who showed abnormalities in frontal white matter also had poorer visuospatial and executive performance, assessed by longer completion times on a set shifting letter-number sequencing task (part B of the Trail Making Test, Warner et al. 2006).

Functional Brain Abnormalities as a Result of Prenatal Cocaine Exposure

To our knowledge, there are three published functional MRI studies of prenatal exposure to cocaine. One perfusion fMRI study reported a $10 \%$ decrease in global cerebral blood flow $(\mathrm{CBF})$ in adolescents with prenatal cocaine exposure. Exposed subjects had reduced global CBF intensities in posterior and inferior brain regions and a 
relative increase in $\mathrm{CBF}$ in anterior and superior brain regions, suggesting that compensatory mechanisms may develop during neural ontogeny (Rao et al. 2007) (Fig. 8). A BOLD fMRI study reported no significant differences in behavioral accuracy or functional brain activation between adolescents with prenatal cocaine exposure and unexposed controls during a nonspatial working memory "N-Back" task, although there was a trend for the non-exposed participants to show greater activation than exposed subjects in the left prefrontal area during the task (Hurt et al. 2008) (Fig. 9). In another BOLD fMRI study of response inhibition, there were no group differences in performance on the Go-No Go task between children with prenatal cocaine exposure and non-exposed controls, but group differences in task-related brain activation were observed. Specifically, the exposed group showed greater activation in right inferior frontal and striatal regions, whereas control subjects showed greater activation in the occipital cortex and fusiform gyrus during the task, suggesting functional differences between groups in neural systems underlying cognitive control and attention regulation (Sheinkopf et al. 2009).

In conclusion, the neuroimaging literature of prenatal exposure to cocaine is much less abundant than that of prenatal exposure to alcohol, and the results published thus far are somewhat inconsistent. Further investigations with large sample sizes will be needed in order to resolve these apparent discrepancies, and improve our understanding of the impact of prenatal cocaine exposure on the developing human brain.

\section{Neuroimaging of Prenatal Exposure to Methamphetamine}

\section{Mechanism of Action and Toxicity}

Methamphetamine acts as a substrate for the plasma membrane dopamine and norepinephrine transporters (DAT and NET); therefore, unlike cocaine, it is transported into cells. Methamphetamine causes reverse transport, whereby the plasma membrane transporters release neurotransmitter molecules into the synapse instead of performing their normal reuptake functions, which leads to a massive increase in synaptic levels of catecholamine neurotransmitters (Sulzer et al. 2005). But methamphetamine also leads to a considerable increase in cytosolic catecholamines via at least three distinct mechanisms. Once inside the nerve terminals, methamphetamine causes the releases of dopamine and norepinephrine from vesicles by blocking the vesicular monoamine transporter (VMAT2). In addition, methamphetamine blocks the catabolism of catecholamines, by inhibiting mitochondrial monoamine oxidase (MAO). Methamphatamine also leads to increased

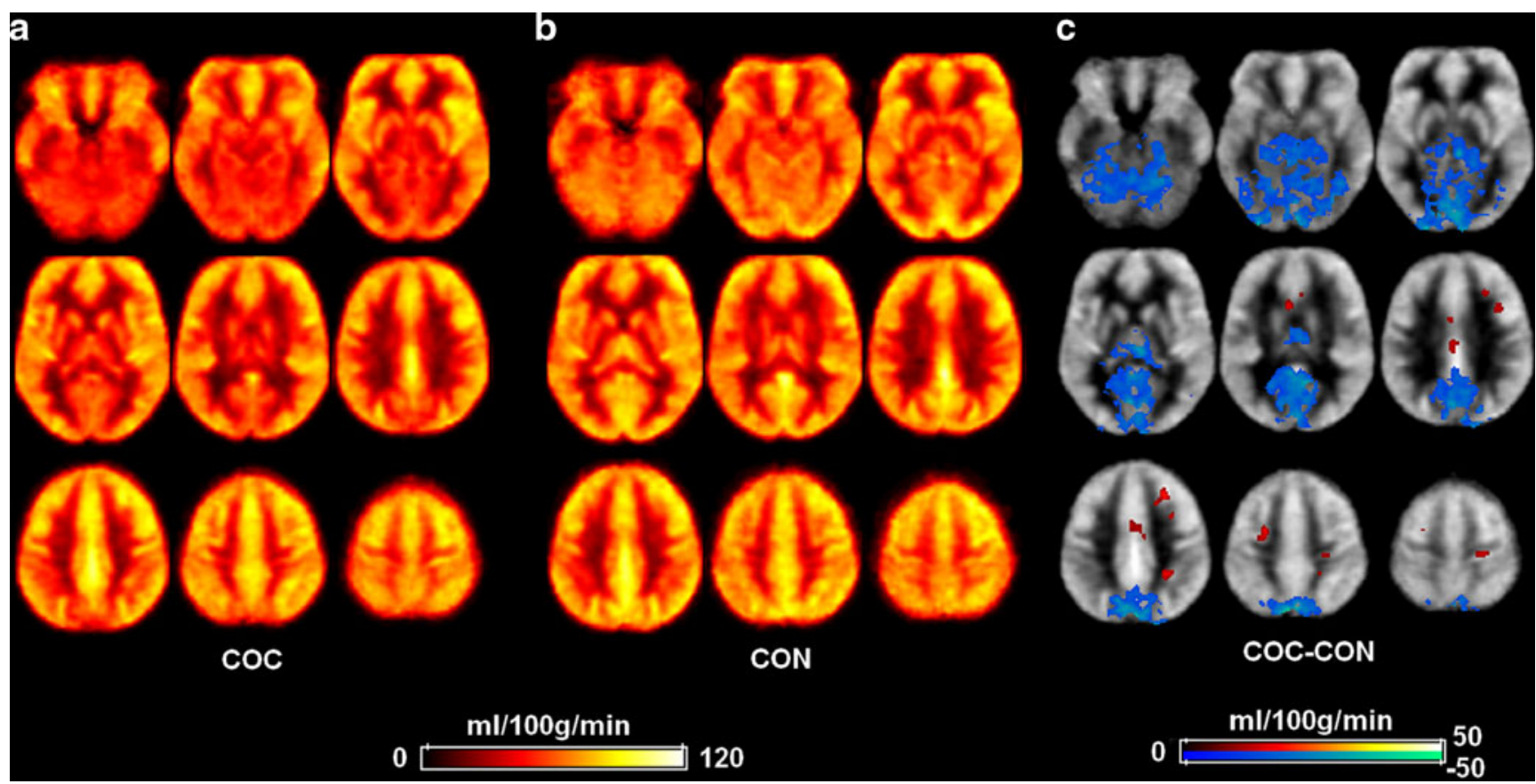

Fig. 8 Quantitative resting $\mathrm{CBF}$ image for the cocaine and control groups. a Mean CBF image for the cocaine group. b Mean CBF image for the control group. $\mathbf{c}$ Quantitative CBF differences between the two groups (cocaine-control; blue color means $\mathrm{CBF}$ is higher for controls, and red color means $\mathrm{CBF}$ is higher for cocaine-exposed subjects). Note the robust CBF decreases in the occipital and thalamus regions for the cocaine group versus the control group (Rao et al. 2007) 
Fig. 9 Areas of activation for functional ROIs (left to right): 1-back minus control condition; 2-back minus control condition; and 2-back minus 1-back. $N=17$ for non-exposed subjects (top), $n=17$ for cocaine-exposed subjects (bottom). There were no significant differences in brain activation between the two groups (Hurt et al. 2008)

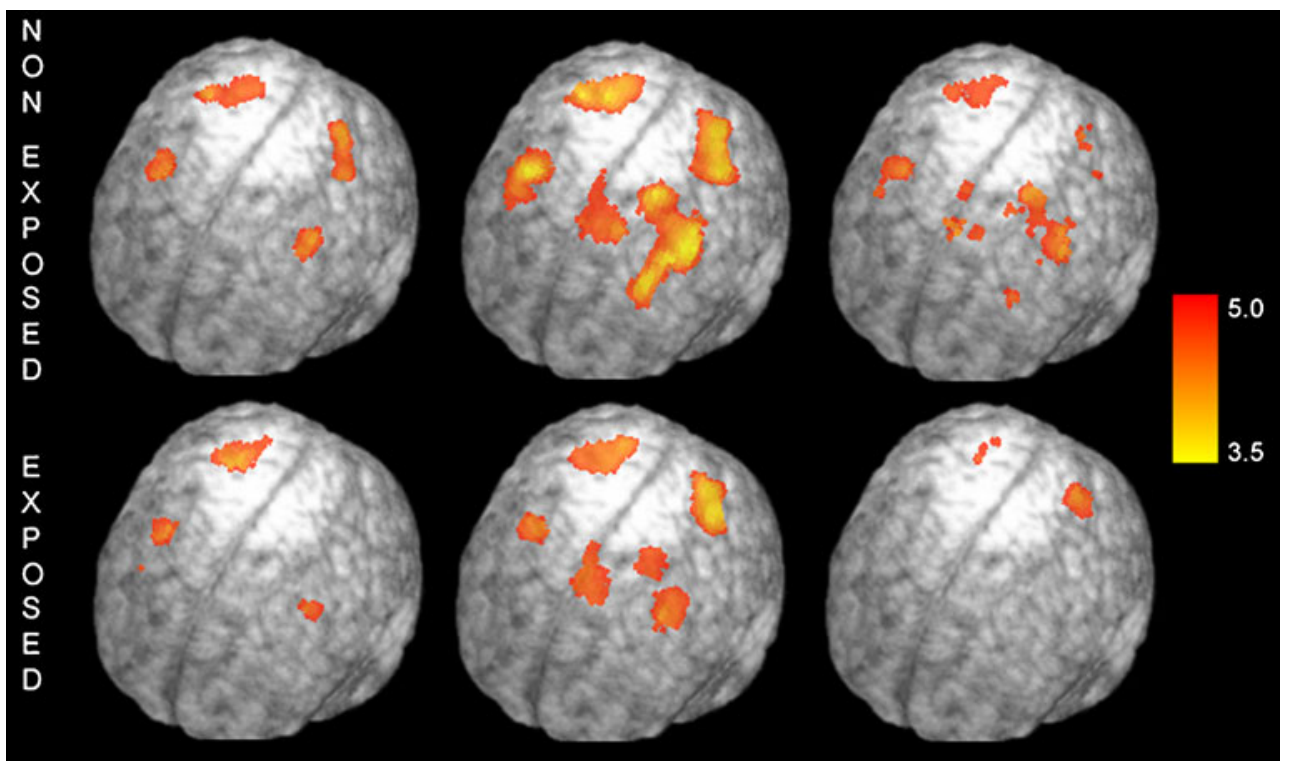

synthesis of dopamine and norepinephrine by stimulating the enzyme tyrosine hydroxylase (Sulzer et al. 2005). Methamphetamine has well-established neurotoxic effects (Frost and Cadet 2000), and the increase in cytosolic catecholamines via the mechanisms described above is believed to underlie most of its neurotoxicity. It leads to the loss of dopamine and norepinephrine terminals because high levels of cytosolic catecholamines are metabolized to reactive metabolites, causing the production of free radicals and leading to oxidative damage. In addition, the production of nitric oxide, p53 activation resulting in apoptosis, excitotoxicity, and mitochondrial damage may also be involved in the neurotoxicity of methamphetamine (Quinton and Yamamoto 2006).

In addition to its direct neurotoxic effects, which have been shown to alter the development of the neural circuitry (Frost and Cadet 2000), methamphetamine may exert significant teratogenic effects on the developing fetus through indirect mechanims, such as vasoconstriction (Burchfield et al. 1991; Stek et al. 1993), potentially resulting in diminished blood flow to the fetus and fetal hypoxia (Stek et al. 1995). Like cocaine, methamphetamine could also alter the developmental trajectory of the fetus by acting on the hypothalamus-pituitary-adrenal axis, disrupting fetal placental monoamine transporter expression, and altering gene expression through epigenetic mechanisms, such as chromatin remodeling and DNA methylation (Salisbury et al. 2009). Rodent models of prenatal exposure to methamphetamine have shown widespread damage to the nervous system, including serotonergic neurotoxic effects and synaptic remodeling of axonal terminals (Weissman and Caldecott-Hazard 1995), disruptions in the levels of monoamine transmitters and altered activity of monoamine systems (Cabrera et al. 1993; Gomes-da-Silva et al. 2002), widespread deficits in the development of the visual system including the retina (Melo, Rodrigues et al. 2006) and the optic nerve (Melo, Moreno et al. 2006), and oxidative DNA damage in the brain, potentially resulting in postnatal motor abnormalities (Jeng et al. 2005).

Structural and Metabolic Brain Abnormalities as a Result of Prenatal Methamphetamine Exposure

Until recently, little was known about the effects of prenatal methamphetamine exposure in humans, but in the last few years, several large prospective studies have reported restricted fetal growth in newborn infants prenatally exposed to methamphetamine (Smith et al. 2006; Nguyen et al. 2010) along with poorer neurobehavioral outcomes, such as under-arousal and depressed movement scores (Smith et al. 2008; Lagasse et al. 2010). Three recent neuroimaging investigations have reported structural brain abnormalities in children with prenatal methamphetamine exposure. A study using sMRI showed that children exposed to methamphetamine in utero had significant regional brain volume reductions in the putamen, globus pallidus, hippocampus, and a trend-level decrease in the volume of the caudate, compared to unexposed controls (Chang et al. 2004). Some of these findings were replicated in another sMRI study aimed at differentiating the effects of prenatal methamphetamine from alcohol exposure. Volume reductions in the striatum, cingulate gyrus, and right inferior frontal gyrus were found to be more severe in children with prenatal exposure to methamphetamine than in subjects with prenatal exposure to alcohol only. These findings suggest that striatal and limbic structures may be especially vulnerable to the teratogenic effects of methamphetamine (Sowell et al. 2010) (Fig. 10). The only DTI study of prenatal methamphetamine exposure reported that exposed children had lower ADC in the frontal and parietal 


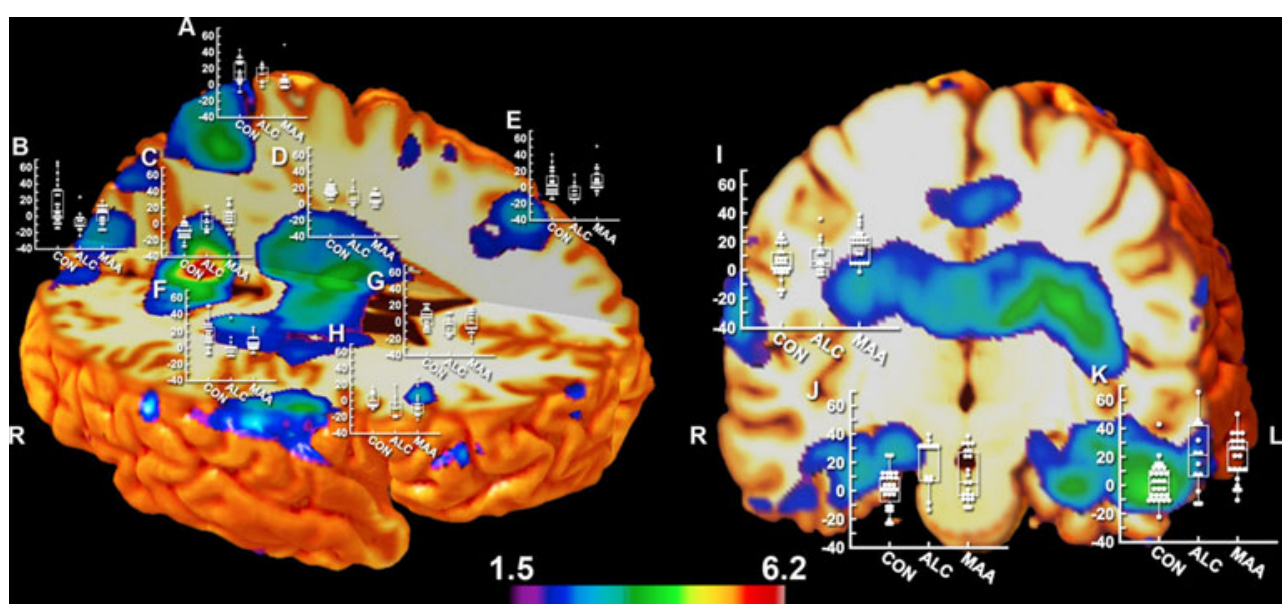

Fig. 10 Statistical $\mathrm{F}$ maps displaying group differences in local brain volume between controls (CON), alcohol-exposed (ALC), and methamphetamine-exposed (MAA) subjects from the three-group analyses. Shown are surface renderings with box plots at various points over the brain where the measurements were taken. The $\mathrm{x}$-axis represents the three groups (CON, ALC, and MAA), and the y-axis represents the percentage (range of -20 to 60 ) of tissue growth and tissue loss in the extracted anatomical points of interest from each

white matter compared to controls, suggesting more compact axons or greater dendritic or spine density (Cloak et al. 2009).

Two MRS studies examined metabolic changes in the brains of children with prenatal exposure to methamphetamine. In one report, exposed children had normal NA concentrations in the frontal white matter and striatum, suggesting no significant neuronal loss in these brain regions. However, they had higher $\mathrm{Cr}$ concentrations than controls in the striatum, which suggests that prenatal methamphetamine exposure results in abnormal energy metabolism in this region (Smith et al. 2001b). In a more recent study by the same group with a larger sample of subjects, metabolic abnormalities were detected in the frontal white matter. Compared to unexposed controls, exposed children had higher tCr, NA, and GLX concentrations in the frontal white matter, suggesting higher neuronal density or cellular compactness in this area. They also had lower $\mathrm{MI}$ and $\mathrm{MI} / \mathrm{tCr}$ ratio in the thalamus, which suggests lower glial content in this structure. Taken together, these findings may indicate aberrant neuronal and glial development in these brain regions (Chang et al. 2009).

Among the studies described above, three have reported correlations between structural or metabolic brain abnormalities and neurocognitive performance in children with prenatal exposure to methamphetamine. Investigators showed that in exposed children, reduced MI concentrations in the thalamus were correlated with poorer performance on a visual motor integration task (Chang et al. 2009). Volume reductions in the putamen, globus pallidus, subject relative to the group mean template of 102 subjects. Shown in color are all voxels in which three-group analyses were statistically significant at a threshold of $p<0.05$ (uncorrected). Anatomical location associated with each graph was as follows: $(A)$ superior parietal lobule; $(B, C)$, supracalcarine cortex; $(D, F)$, thalamus; $(E)$, midcingulate; $(G, H)$, caudate; $(I)$, posterior inferior frontal gyrus; $(J, K)$, temporal fusiform cortex (Sowell et al. 2010)

and hippocampus were found to be correlated with poorer performance on sustained attention and delayed verbal memory in children exposed to methamphetamine in utero (Chang et al. 2004). Striatal abnormalities were also shown to be associated cognitive deficit in another study. In children with prenatal methamphetamine exposure, volume reductions in the caudate were reported to correlate with full-scale intelligence quotient (FSIQ) scores (Sowell et al. 2010).

\section{Functional Brain Abnormalities as a Result of Prenatal Methamphetamine Exposure}

To our knowledge, only one fMRI investigation of prenatal methamphetamine exposure has been published thus far. This study, which relied on a sample of subjects with prenatal exposure to methamphetamine and alcohol overlapping with the samples used in one of the sMRI studies described above (Sowell et al. 2010), examined functional brain activation during a verbal learning and memory task. Investigators reported that children with prenatal exposure to methamphetamine (most of whom had concomitant alcohol exposure) activated more diffuse brain regions, including bilateral medial temporal structures known to be important for memory, than both children with prenatal exposure to alcohol alone and unexposed controls. These findings might indicate that children with prenatal methamphetamine exposure have to recruit compensatory systems to support a weak verbal memory network. Although explanations for activation differences remain speculative, these results may suggest that prenatal meth- 
amphetamine exposure affects brain networks involved in verbal memory above and beyond the effects of prenatal exposure to alcohol alone (Lu et al. 2009) (Fig. 11).

In summary, very few neuroimaging studies of prenatal exposure to methamphetamine have been published so far; thus, it may be too early to draw specific conclusions as to which brain systems are most affected by prenatal exposure to this drug in humans. The only trend that is beginning to emerge from the current literature is that methamphetamine may be particularly toxic to the striatum, which makes sense in the context of its mechanism of action on catecholamine transmitters and its known toxicity to dopamine terminals. Further investigations will be needed in order to obtain a better understanding of the impact of prenatal exposure to methamphetamine on the developing nervous system in humans.

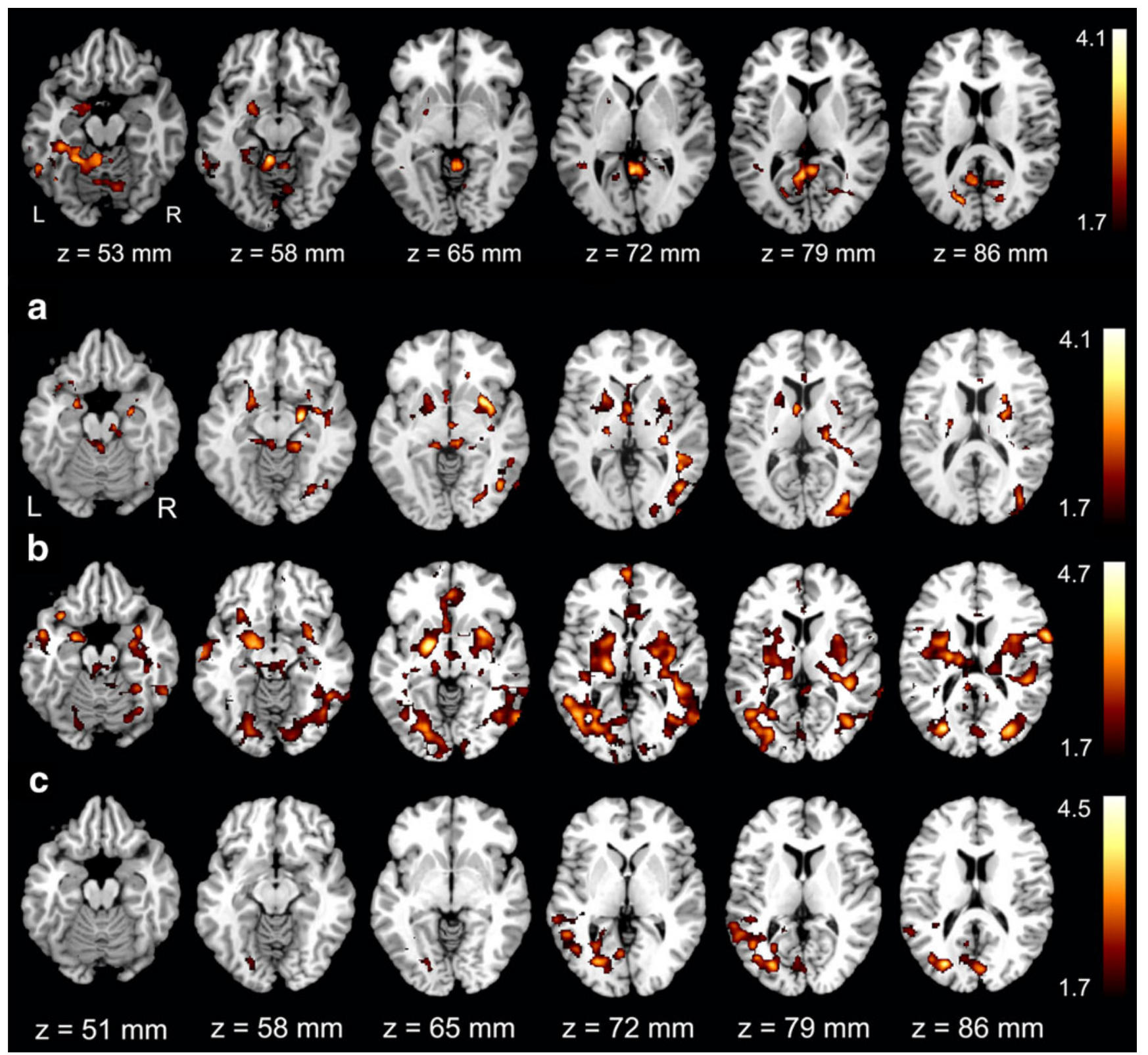

Fig. 11 Top Panel: Correlation between performance and activation was significant in left medial temporal structures for the control group only. The color bar codes for $\mathrm{Z}$ statistics of significant clusters thresholded at $p \leq 0.05$. Z axis values on the bottom correspond to MNI standard space coordinates. Bottom panel: Group differences in the (Learning+Recall)-Rest contrast with IQ regressed out. a MA $>\mathrm{CON}$, b MA>ALC, $\mathbf{c} C \mathrm{CON}>\mathrm{ALC}$. The color bars on the right code for $Z$ statistics of significant clusters thresholded at $p \leq 0.05$. $Z$ axis values on the bottom correspond to MNI standard space coordinates. MA = Methamphetamine-exposed group, ALC $=$ Alcohol-exposed group, $\mathrm{CON}=$ unexposed control group ( $\mathrm{Lu}$ et al. 2009) 


\section{Conclusions}

As reviewed above, recent advances in neuroimaging techniques have helped improve our understanding of the effects of prenatal exposure to drugs of abuse on the developing human brain. However, certain drugs have been more studied than others, with most of the published literature reporting on the effects of prenatal alcohol exposure. When reviewing studies examining structural and metabolic changes in the brains of children with FASD, it appears that most brain systems are affected by the teratogenic consequences of prenatal alcohol exposure. Although early reports suggested that gross lobar regions of the brain such as the frontal and temporal lobes might be relatively spared relative to overall brain volume reduction (Archibald et al. 2001), more recent studies with enhanced spatial sensitivity suggest that these areas are also impacted, with children with FASD showing increased cortical thickness in some parts of the frontal and temporal lobes (Sowell et al. 2008a). The fMRI studies published so far suggest that subjects with heavy prenatal alcohol exposure show abnormal patterns of brain activation in many cognitive domains. Future neuroimaging studies should aim at replicating these findings in larger samples of subjects and with different methods.

The body of literature about the effects of prenatal exposure to illegal stimulant drugs is much smaller. The studies that examined the effects of prenatal exposure to cocaine have yielded inconsistent results, with some reporting regional brain volume reductions (Dow-Edwards et al. 2006) and others suggesting the absence of such volume decreases (Smith et al. 2001a), although two separate studies indicate that the striatum may be particularly affected (Rao et al. 2007; Avants et al. 2007). Results from fMRI studies are also inconclusive, with a study reporting the absence of significant differences in brain activation between exposed and non-exposed subjects (Hurt et al. 2008), and another investigation suggesting the existence of group differences in brain activation while performing a different task (Sheinkopf et al. 2009). The published studies about the effects of prenatal exposure to methamphetamine have provided more consistent findings, and suggest the existence of structural and metabolic brain abnormalities in children exposed to methamphetamine in utero in several brain regions, especially in the striatum (Chang et al. 2004; Smith et al. 2001b). Evidence for the clinical relevance of these abnormalities is also beginning to emerge (Sowell et al. 2010). Little can be said about functional brain abnormalities, however, since only one fMRI study of prenatal methamphetamine exposure has been published so far. Clearly, the study of prenatal exposure to illegal stimulants with neuroimaging is still in its infancy and further investigations with large sample sizes will be needed in order to confirm the current findings about methamphetamine and to better interpret the presently conflicting results about cocaine.

One important limitation to keep in mind when reviewing human studies of prenatal exposure to drugs of abuse is the problem of polysubstance exposure. For instance, a recent study showed that almost half of women who use methamphetamine during pregnancy also use alcohol (Smith et al. 2006). Thus, future studies should try to isolate the effects of individual drugs, and one way to achieve this is to use large samples of subjects. Large sample sizes provide more statistical power and may allow for investigators to covary the effects of a drug of interest for the effects of other drugs, provided that they have enough information about what particular combinations of drugs were used. The problem of concurrent exposure to nicotine is of particular interest here, because tobacco is often used in conjunction with alcohol and stimulants, and nicotine has been shown to induce lasting abnormalities in neurogenesis in animal models (Slotkin 1998).

The issue of polydrug exposure is further complicated by the fact that most current human studies of prenatal exposure are retrospective. Thus, accurate information of what combinations of drugs were used, in what quantities, and at what points during pregnancy is often unavailable. This is because many subjects in these studies have been removed from their biological families because of the presence of drugs of abuse in the home. Although social, medical, and legal records are often used in addition to reports from adoptive parents, it is often hard to determine precise exposure histories for these subjects. This is also the case with subjects who are still under the custody of their biological mothers, because frequencies of drug exposure are difficult to accurately recall years after the drug use, and may be compounded by the stigma of admitting to drug use during pregnancy. In order to remedy this problem, future investigations should rely on prospectively enrolled subjects, for whom more accurate exposure histories and dosages can be obtained.

Finally, future studies should aim at elucidating the relationships between structural, metabolic, and functional abnormalities and children's actual challenges outside the scanner. For instance, in the case of prenatal alcohol exposure, future investigations should examine possible relationships between brain abnormalities and facial dysmorphology. For all drugs of abuse, relationships between brain and behavior should be further examined. Most existing studies have attempted to investigate brainbehavior relationships through correlational methods on a structure-by-structure basis. Future studies may benefit from integrating observations from different brain imaging modalities and investigating brain structure, function, and connectivity in the same children, in order to determine how these changes relate to cognitive function in various 
domains, but most importantly to children's behaviors in the "real world." Although the interpretation of findings from such studies will be limited by the nature of correlational research, such investigations will provide important insights that may ultimately help clinicians devise appropriate interventions in order to address the specific needs of children with prenatal exposure to drugs of abuse.

Acknowledgements All or part of this work was done in conjunction with the Collaborative Initiative on Fetal Alcohol Spectrum Disorders (CIFASD), which is funded by grants from the National Institute on Alcohol and Alcohol Abuse (NIAAA). Additional information about CIFASD can be found at www.cifasd.org. This work was also supported by NIDA R01 DA017831, NIAAA U01 AA017122-01, NICHD R01 HD053893-01, and the March of Dimes 6-FY2008-50 (ERS PI), and NCRR grants U54 RR021813 entitled Center for Computational Biology (CCB), and P41 RR013642 (PI A.W. Toga)

Open Access This article is distributed under the terms of the Creative Commons Attribution Noncommercial License which permits any noncommercial use, distribution, and reproduction in any medium, provided the original author(s) and source are credited.

\section{References}

Archibald, S. L., Fennema-Notestine, C., Gamst, A., Riley, E. P., Mattson, S. N., \& Jernigan, T. L. (2001). Brain dysmorphology in individuals with severe prenatal alcohol exposure. Developmental Medicine and Child Neurology, 43(3), 148-154.

Astley, S. J., \& Clarren, S. K. (2000). Diagnosing the full spectrum of fetal alcohol-exposed individuals: introducing the 4-digit diagnostic code. Alcohol and Alcoholism, 35(4), 400-410.

Astley, S. J., Richards, T., Aylward, E. H., Olson, H. C., Kerns, K., Brooks, A., et al. (2009a). Magnetic resonance spectroscopy outcomes from a comprehensive magnetic resonance study of children with fetal alcohol spectrum disorders. Magnetic Resonance Imaging, 27(6), 760-778.

Astley, S. J., Aylward, E. H., Carmichael Olson, H., et al. (2009b). Functional magnetic resonance imaging outcomes from a comprehensive magnetic resonance study of children with fetal alcohol spectrum disorders. Journal of Neurodevelopmental Disorders, 1(1), 61-80.

Avants, B. B., Hurt, H., Giannetta, J. M., Epstein, C. L., Shera, D. M., Rao, H., et al. (2007). Effects of heavy in utero cocaine exposure on adolescent caudate morphology. Pediatric Neurology, 37(4), 275-279.

Barrow Heaton, M. B., Kidd, K., Bradley, D., Paiva, M., Mitchell, J., \& Walker, D. W. (1999). Prenatal ethanol exposure reduces spinal cord motoneuron number in the fetal rat but does not affect GDNF target tissue protein. Developmental Neuroscience, 21(6), $444-452$.

Basser, P. J., Mattiello, J., \& LeBihan, D. (1994). MR diffusion tensor spectroscopy and imaging. Biophysical Journal, 66(1), 259-267. PMCID: 1275686.

Bhat, R. V., \& Baraban, J. M. (1993). Activation of transcription factor genes in striatum by cocaine: role of both serotonin and dopamine systems. The Journal of Pharmacology and Experimental Therapeutics, 267(1), 496-505.

Bookstein, F. L., Sampson, P. D., Streissguth, A. P., \& Connor, P. D. (2001). Geometric morphometrics of corpus callosum and subcortical structures in the fetal-alcohol-affected brain. Teratology, 64(1), 4-32.

Bookstein, F. L., Streissguth, A. P., Sampson, P. D., Connor, P. D., \& Barr, H. M. (2002). Corpus callosum shape and neuropsychological deficits in adult males with heavy fetal alcohol exposure. Neuroimage, 15(1), 233-251.

Borodinsky, L. N., O'Leary, D., Neale, J. H., Vicini, S., Coso, O. A., \& Fiszman, M. L. (2003). GABA-induced neurite outgrowth of cerebellar granule cells is mediated by $\operatorname{GABA}(\mathrm{A})$ receptor activation, calcium influx and CaMKII and erk $1 / 2$ pathways. Journal of Neurochemistry, 84(6), 1411-1420.

Burchfield, D. J., Lucas, V. W., Abrams, R. M., Miller, R. L., \& DeVane, C. L. (1991). Disposition and pharmacodynamics of methamphetamine in pregnant sheep. JAMA, 265(15), 1968-1973.

Cabrera, T. M., Levy, A. D., Li, Q., van de Kar, L. D., \& Battaglia, G. (1993). Prenatal methamphetamine attenuates serotonin mediated renin secretion in male and female rat progeny: evidence for selective long-term dysfunction of serotonin pathways in brain. Synapse, 15(3), 198-208.

Chang, L., Smith, L. M., LoPresti, C., Yonekura, M. L., Kuo, J., Walot, I., et al. (2004). Smaller subcortical volumes and cognitive deficits in children with prenatal methamphetamine exposure. Psychiatry Research, 132(2), 95-106.

Chang, L., Cloak, C., Jiang, C. S., Farnham, S., Tokeshi, B., Buchthal, S., et al. (2009). Altered neurometabolites and motor integration in children exposed to methamphetamine in utero. Neuroimage, 48(2), 391-397.

Clarren, S. K., \& Smith, D. W. (1978). The fetal alcohol syndrome. The Lamp, 35(10), 4-7.

Cloak, C. C., Ernst, T., Fujii, L., Hedemark, B., \& Chang, L. (2009). Lower diffusion in white matter of children with prenatal methamphetamine exposure. Neurology, 72(24), 2068-2075. PMCID: 2697962.

Cohen, M. S., \& Bookheimer, S. Y. (1994). Localization of brain function using magnetic resonance imaging. Trends in Neurosciences, 17(7), 268-277.

Cohen, H. L., Sloves, J. H., Laungani, S., Glass, L., \& DeMarinis, P. (1994). Neurosonographic findings in full-term infants born to maternal cocaine abusers: visualization of subependymal and periventricular cysts. Journal of Clinical Ultrasound, 22(5), 327333.

Cortese, B. M., Moore, G. J., Bailey, B. A., Jacobson, S. W., DelaneyBlack, V., \& Hannigan, J. H. (2006). Magnetic resonance and spectroscopic imaging in prenatal alcohol-exposed children: preliminary findings in the caudate nucleus. Neurotoxicology and Teratology, 28(5), 597-606.

Crandall, J. E., Hackett, H. E., Tobet, S. A., Kosofsky, B. E., \& Bhide, P. G. (2004). Cocaine exposure decreases GABA neuron migration from the ganglionic eminence to the cerebral cortex in embryonic mice. Cerebral Cortex, 14(6), 665-675. PMCID: 1224742.

Detre, J. A., Leigh, J. S., Williams, D. S., \& Koretsky, A. P. (1992). Perfusion imaging. Magnetic Resonance in Medicine, 23(1), 37-45.

Dodge, N. C., Jacobson, J. L., Molteno, C. D., Meintjes, E. M., Bangalore, S., Diwadkar, V., et al. (2009). Prenatal alcohol exposure and interhemispheric transfer of tactile information: Detroit and Cape Town findings. Alcohol Clin Exp Res, 33(9), 1628-1637. PMCID: 2768060.

Dow-Edwards, D. L., Benveniste, H., Behnke, M., Bandstra, E. S., Singer, L. T., Hurd, Y. L., et al. (2006). Neuroimaging of prenatal drug exposure. Neurotoxicology and Teratology, 28(3), 386-402.

Fagerlund, A., Heikkinen, S., Autti-Ramo, I., Korkman, M., Timonen, M., Kuusi, T., et al. (2006). Brain metabolic alterations in adolescents and young adults with fetal alcohol spectrum disorders. Alcoholism, Clinical and Experimental Research, 30 (12), 2097-2104. 
Feng, M. J., Yan, S. E., \& Yan, Q. S. (2005). Effects of prenatal alcohol exposure on brain-derived neurotrophic factor and its receptor tyrosine kinase B in offspring. Brain Research, 1042(2), 125-132.

Friedman, E., Yadin, E., \& Wang, H. Y. (1996). Effect of prenatal cocaine on dopamine receptor-G protein coupling in mesocortical regions of the rabbit brain. Neuroscience, 70(3), 739-747.

Frost, D. O., \& Cadet, J. L. (2000). Effects of methamphetamineinduced neurotoxicity on the development of neural circuitry: a hypothesis. Brain Research. Brain Research Reviews, 34(3), $103-118$.

Fryer, S. L., Tapert, S. F., Mattson, S. N., Paulus, M. P., Spadoni, A. D., \& Riley, E. P. (2007). Prenatal alcohol exposure affects frontal-striatal BOLD response during inhibitory control. Alcoholism, Clinical and Experimental Research, 31(8), 1415-1424.

Fryer, S. L., Schweinsburg, B. C., Bjorkquist, O. A., Frank, L. R., Mattson, S. N., Spadoni, A. D., et al. (2009). Characterization of white matter microstructure in fetal alcohol spectrum disorders. Alcohol Clin Exp Res, 33(3), 514-521. PMCID: 2651988.

Gieron-Korthals, M. A., Helal, A., \& Martinez, C. R. (1994). Expanding spectrum of cocaine induced central nervous system malformations. Brain \& Development, 16(3), 253-256.

Glavas, M. M., Ellis, L., Yu, W. K., \& Weinberg, J. (2007). Effects of prenatal ethanol exposure on basal limbic-hypothalamicpituitary-adrenal regulation: role of corticosterone. Alcoholism, Clinical and Experimental Research, 31(9), 1598-1610.

Gomes-da-Silva, J., Perez-Rosado, A., de Miguel, R., Fernandez-Ruiz, J., Silva, M. C., \& Tavares, M. A. (2002). Prenatal exposure to methamphetamine in the rat: ontogeny of tyrosine hydroxylase mRNA expression in mesencephalic dopaminergic neurons. Annals of the New York Academy of Sciences, 965, 68-77.

Gomez-Anson, B., \& Ramsey, R. G. (1994). Pachygyria in a neonate with prenatal cocaine exposure: MR features. Journal of Computer Assisted Tomography, 18(4), 637-639.

Gressens, P., Kosofsky, B. E., \& Evrard, P. (1992). Cocaine-induced disturbances of corticogenesis in the developing murine brain. Neuroscience Letters, 140(1), 113-116.

Guerriero, R. M., Rajadhyaksha, A., Crozatier, C., Giros, B., NostenBertrand, M., \& Kosofsky, B. E. (2005). Augmented constitutive CREB expression in the nucleus accumbens and striatum may contribute to the altered behavioral response to cocaine of adult mice exposed to cocaine in utero. Developmental Neuroscience, 27(2-4), 235-248.

Heier, L. A., Carpanzano, C. R., Mast, J., Brill, P. W., Winchester, P., \& Deck, M. D. (1991). Maternal cocaine abuse: the spectrum of radiologic abnormalities in the neonatal CNS. AJNR. American Journal of Neuroradiology, 12(5), 951-956.

Hendee, W. R., \& Morgan, C. J. (1984). Magnetic resonance imaging. Part I-physical principles. The Western Journal of Medicine, 141 (4), 491-500. PMCID: 1021860.

Hoffman, P. L., Rabe, C. S., Moses, F., \& Tabakoff, B. (1989). Nmethyl-D-aspartate receptors and ethanol: inhibition of calcium flux and cyclic GMP production. Journal of Neurochemistry, 52 (6), 1937-1940.

Honse, Y., Nixon, K. M., Browning, M. D., \& Leslie, S. W. (2003). Cell surface expression of NR1 splice variants and NR2 subunits is modified by prenatal ethanol exposure. Neuroscience, 122(3), 689-698.

Hughes, P. D., Wilson, W. R., \& Leslie, S. W. (2001). Effect of gestational ethanol exposure on the NMDA receptor complex in rat forebrain: from gene transcription to cell surface. Brain Research. Developmental Brain Research, 129(2), 135145 .

Hurt, H., Giannetta, J. M., Korczykowski, M., Hoang, A., Tang, K. Z., Betancourt, L., et al. (2008). Functional magnetic resonance imaging and working memory in adolescents with gestational cocaine exposure. Jornal de Pediatria, 152(3), 371-377.
Imperato, A., \& Di Chiara, G. (1986). Preferential stimulation of dopamine release in the nucleus accumbens of freely moving rats by ethanol. The Journal of Pharmacology and Experimental Therapeutics, 239(1), 219-228.

Jeng, W., Wong, A. W., Ting, A. K. R., \& Wells, P. G. (2005). Methamphetamine-enhanced embryonic oxidative DNA damage and neurodevelopmental deficits. Free Radical Biology \& Medicine, 39(3), 317-326.

Jones, K. L., \& Smith, D. W. (1973). Recognition of the fetal alcohol syndrome in early infancy. Lancet, 302(7836), 999-1001.

Jones, K. L., Smith, D. W., Ulleland, C. N., \& Streissguth, P. (1973). Pattern of malformation in offspring of chronic alcoholic mothers. Lancet, 1(7815), 1267-1271.

Jones, L. B., Stanwood, G. D., Reinoso, B. S., Washington, R. A., Wang, H. Y., Friedman, E., et al. (2000). In utero cocaineinduced dysfunction of dopamine D1 receptor signaling and abnormal differentiation of cerebral cortical neurons. The Journal of Neuroscience, 20(12), 4606-4614.

Koegler, S. M., Seidler, F. J., Spencer, J. R., \& Slotkin, T. A. (1991). Ischemia contributes to adverse effects of cocaine on brain development: suppression of ornithine decarboxylase activity in neonatal rat. Brain Research Bulletin, 27(6), 829-834.

Kubrusly, R. C., \& Bhide, P. G. (2010). Cocaine exposure modulates dopamine and adenosine signaling in the fetal brain. Neuropharmacology, 58(2), 436-443. PMCID: 2813374.

Lagasse, L. L., Wouldes, T., Newman, E., Smith, L. M., Shah, R. Z., Derauf, C., et al (2010). Prenatal methamphetamine exposure and neonatal neurobehavioral outcome in the USA and New Zealand. Neurotoxicology and Teratology. doi:10.1016/j.ntt.2010.06.009.

Le Bihan, D. (1995). Molecular diffusion, tissue microdynamics and microstructure. NMR in Biomedicine, 8(7-8), 375-386.

Lebel, C., Rasmussen, C., Wyper, K., Walker, L., Andrew, G., Yager, J., et al. (2008). Brain diffusion abnormalities in children with fetal alcohol spectrum disorder. Alcoholism, Clinical and Experimental Research, 32(10), 1732-1740.

Lebel, C., Rasmussen, C., Wyper, K., Andrew, G., \& Beaulieu, C. (2010). Brain microstructure is related to math ability in children with fetal alcohol spectrum disorder. Alcoholism, Clinical and Experimental Research, 34(2), 354-363.

Lester, B. M., \& Padbury, J. F. (2009). Third pathophysiology of prenatal cocaine exposure. Developmental Neuroscience, 31(12), 23-35.

Levitt, P., Harvey, J. A., Friedman, E., Simansky, K., \& Murphy, E. H. (1997). New evidence for neurotransmitter influences on brain development. Trends in Neurosciences, 20(6), 269-274.

Li, L., Coles, C. D., Lynch, M. E., \& Hu, X. (2009). Voxelwise and skeleton-based region of interest analysis of fetal alcohol syndrome and fetal alcohol spectrum disorders in young adults. Human Brain Mapping, 30(10), 3265-3274. PMCID: 2748153.

Lidow, M. S. (1995). Prenatal cocaine exposure adversely affects development of the primate cerebral cortex. Synapse, 21(4), 332341.

Lidow, M. S., \& Song, Z. M. (2001a). Effect of cocaine on cell proliferation in the cerebral wall of monkey fetuses. Cerebral Cortex, 11(6), 545-551.

Lidow, M. S., \& Song, Z. M. (2001b). Primates exposed to cocaine in utero display reduced density and number of cerebral cortical neurons. The Journal of Comparative Neurology, 435(3), 263275.

Lu, L. H., Johnson, A., O'Hare, E. D., Bookheimer, S. Y., Smith, L. M., O'Connor, M. J., et al. (2009). Effects of prenatal methamphetamine exposure on verbal memory revealed with functional magnetic resonance imaging. Journal of Developmental and Behavioral Pediatrics, 30(3), 185-192. PMCID: 2745202. 
Ma, X., Coles, C. D., Lynch, M. E., Laconte, S. M., Zurkiya, O., Wang, D., et al. (2005). Evaluation of corpus callosum anisotropy in young adults with fetal alcohol syndrome according to diffusion tensor imaging. Alcoholism, Clinical and Experimental Research, 29(7), 1214-1222.

Malanga, C. J., 3rd, \& Kosofsky, B. E. (1999). Mechanisms of action of drugs of abuse on the developing fetal brain. Clinicas de Perinatologia, 26(1), 17-37. v-vi.

Malisza, K. L., Allman, A. A., Shiloff, D., Jakobson, L., Longstaffe, S., \& Chudley, A. E. (2005). Evaluation of spatial working memory function in children and adults with fetal alcohol spectrum disorders: a functional magnetic resonance imaging study. Pediatric Research, 58(6), 1150-1157.

Mattson, S. N., \& Riley, E. P. (1998). A review of the neurobehavioral deficits in children with fetal alcohol syndrome or prenatal exposure to alcohol. Alcoholism, Clinical and Experimental Research, 22(2), 279-294.

Mattson, S. N., Riley, E. P., Sowell, E. R., Jernigan, T. L., Sobel, D. F., \& Jones, K. L. (1996). A decrease in the size of the basal ganglia in children with fetal alcohol syndrome. Alcoholism, Clinical and Experimental Research, 20(6), 1088-1093.

Mayes, L. C. (1999). Developing brain and in utero cocaine exposure: effects on neural ontogeny. Development and Psychopathology, $11(4), 685-714$.

Melo, P., Rodrigues, L. G., Silva, M. C., \& Tavares, M. A. (2006a). Effects of prenatal exposure to methamphetamine on the development of the rat retina. Annals of the New York Academy of Sciences, 1074, 590-603.

Melo, P., Moreno, V. Z., Vazquez, S. P., Pinazo-Duran, M. D., \& Tavares, M. A. (2006b). Myelination changes in the rat optic nerve after prenatal exposure to methamphetamine. Brain Research, 1106(1), 21-29.

Meyer, J. S., Shearman, L. P., Collins, L. M., \& Maguire, R. L. (1993). Cocaine binding sites in fetal rat brain: implications for prenatal cocaine action. Psychopharmacology (Berl), 112(4), 445-451.

Miller, M. W. (2003). Expression of transforming growth factor-beta in developing rat cerebral cortex: effects of prenatal exposure to ethanol. The Journal of Comparative Neurology, 460(3), 410 424

Miller, M. W., \& Dow-Edwards, D. L. (1988). Structural and metabolic alterations in rat cerebral cortex induced by prenatal exposure to ethanol. Brain Research, 474(2), 316-326.

Miller, M. W., \& Robertson, S. (1993). Prenatal exposure to ethanol alters the postnatal development and transformation of radial glia to astrocytes in the cortex. The Journal of Comparative Neurology, 337(2), 253-266.

Mitchell, J. J., Paiva, M., Moore, D. B., Walker, D. W., \& Heaton, M. B. (1998). A comparative study of ethanol, hypoglycemia, hypoxia and neurotrophic factor interactions with fetal rat hippocampal neurons: a multi-factor in vitro model developmental ethanol effects. Brain Research. Developmental Brain Research, 105(2), 241-250.

Nguyen, D., Smith, L. M., Lagasse, L. L., Derauf, C., Grant, P., Shah, R., et al. (2010). Intrauterine growth of infants exposed to prenatal methamphetamine: results from the infant development, environment, and lifestyle study. Jornal de Pediatria, 157(2), 337-339.

Novikova, S. I., He, F., Bai, J., Badan, I., Lidow, I. A., \& Lidow, M. S. (2005). Cocaine-induced changes in the expression of apoptosis-related genes in the fetal mouse cerebral wall. Neurotoxicology and Teratology, 27(1), 3-14.

O'Hare, E. D., Kan, E., Yoshii, J., Mattson, S. N., Riley, E. P., Thompson, P. M., et al. (2005). Mapping cerebellar vermal morphology and cognitive correlates in prenatal alcohol exposure. NeuroReport, 16(12), 1285-1290.

O'Hare, E. D., Lu, L. H., Houston, S. M., Bookheimer, S. Y., Mattson, S. N., O'Connor, M. J., et al. (2009). Altered frontal-parietal functioning during verbal working memory in children and adolescents with heavy prenatal alcohol exposure. Human Brain Mapping, 30(10), 3200-3208. PMCID: 2748151.

Olney, J. W., Ishimaru, M. J., Bittigau, P., \& Ikonomidou, C. (2000). Ethanol-induced apoptotic neurodegeneration in the developing brain. Apoptosis, 5(6), 515-521.

Ornoy, A. (2007). Embryonic oxidative stress as a mechanism of teratogenesis with special emphasis on diabetic embryopathy. Reproductive Toxicology, 24(1), 31-41.

Ozer, E., Sarioglu, S., \& Gure, A. (2000). Effects of prenatal ethanol exposure on neuronal migration, neuronogenesis and brain myelination in the mice brain. Clinical Neuropathology, 19(1), $21-25$.

Pollard, I. (2007). Neuropharmacology of drugs and alcohol in mother and fetus. Seminars in Fetal \& Neonatal Medicine, 12(2), 106-113.

Quinton, M. S., \& Yamamoto, B. K. (2006). Causes and consequences of methamphetamine and MDMA toxicity. The AAPS Journal, 8 (2), E337-E347.

Rao, H., Wang, J., Giannetta, J., Korczykowski, M., Shera, D., Avants, B. B., et al. (2007). Altered resting cerebral blood flow in adolescents with in utero cocaine exposure revealed by perfusion functional MRI. Pediatrics, 120(5), e1245-e1254.

Redila, V. A., Olson, A. K., Swann, S. E., Mohades, G., Webber, A. J., Weinberg, J., et al. (2006). Hippocampal cell proliferation is reduced following prenatal ethanol exposure but can be rescued with voluntary exercise. Hippocampus, 16(3), 305-311.

Ren, J. Q., Malanga, C. J., Tabit, E., \& Kosofsky, B. E. (2004). Neuropathological consequences of prenatal cocaine exposure in the mouse. International Journal of Developmental Neuroscience, 22(5-6), 309-320. PMCID: 2664265.

Riley, E. P., Mattson, S. N., Sowell, E. R., Jernigan, T. L., Sobel, D. F., \& Jones, K. L. (1995). Abnormalities of the corpus callosum in children prenatally exposed to alcohol. Alcoholism, Clinical and Experimental Research, 19(5), 1198-1202.

Ritz, M. C., Cone, E. J., \& Kuhar, M. J. (1990). Cocaine inhibition of ligand binding at dopamine, norepinephrine and serotonin transporters: a structure-activity study. Life Sciences, 46(9), 635-645.

Rivkin, M. J., Davis, P. E., Lemaster, J. L., Cabral, H. J., Warfield, S. K., Mulkern, R. V., et al. (2008). Volumetric MRI study of brain in children with intrauterine exposure to cocaine, alcohol, tobacco, and marijuana. Pediatrics, 121(4), 741-750. PMCID: 2562785.

Ross, B., \& Bluml, S. (2001). Magnetic resonance spectroscopy of the human brain. The Anatomical Record, 265(2), 54-84.

Salisbury, A. L., Ponder, K. L., Padbury, J. F., \& Lester, B. M. (2009). Fetal effects of psychoactive drugs. Clinics in Perinatology, 36 (3), 595-619. PMCID: 2767264.

Schenker, S., Yang, Y., Johnson, R. F., Downing, J. W., Schenken, R. S., Henderson, G. I., et al. (1993). The transfer of cocaine and its metabolites across the term human placenta. Clinical Pharmacology and Therapeutics, 53(3), 329-339.

Schwartz, M. L., \& Meinecke, D. L. (1992). Early expression of GABA-containing neurons in the prefrontal and visual cortices of rhesus monkeys. Cerebral Cortex, 2(1), 16-37.

Sheinkopf, S. J., Lester, B. M., Sanes, J. N., Eliassen, J. C., Hutchison, E. R., Seifer, R., et al. (2009). Functional MRI and response inhibition in children exposed to cocaine in utero. Preliminary findings. Developmental Neuroscience, 31(1-2), 159-166.

Shetty, A. K., \& Phillips, D. E. (1992). Effects of prenatal ethanol exposure on the development of Bergmann glia and astrocytes in the rat cerebellum: an immunohistochemical study. The Journal of Comparative Neurology, 321(1), 19-32.

Sliwowska, J. H., Barker, J. M., Barha, C. K., Lan, N., Weinberg, J., \& Galea, L. A. (2010). Stress-induced suppression of hippocampal neurogenesis in adult male rats is altered by prenatal ethanol exposure. Stress, 13(4), 301-313. 
Slotkin, T. A. (1998). Fetal nicotine or cocaine exposure: which one is worse? The Journal of Pharmacology and Experimental Therapeutics, 285(3), 931-945.

Smith, L. M., Chang, L., Yonekura, M. L., Gilbride, K., Kuo, J., Poland, R. E., et al. (2001a). Brain proton magnetic resonance spectroscopy and imaging in children exposed to cocaine in utero. Pediatrics, 107(2), 227-231.

Smith, L. M., Chang, L., Yonekura, M. L., Grob, C., Osborn, D., \& Ernst, T. (2001b). Brain proton magnetic resonance spectroscopy in children exposed to methamphetamine in utero. Neurology, 57(2), 255-260.

Smith, L. M., LaGasse, L. L., Derauf, C., Grant, P., Shah, R., Arria, A., et al. (2006). The infant development, environment, and lifestyle study: effects of prenatal methamphetamine exposure, polydrug exposure, and poverty on intrauterine growth. Pediatrics, 118(3), 1149-1156.

Smith, L. M., Lagasse, L. L., Derauf, C., Grant, P., Shah, R., Arria, A., et al. (2008). Prenatal methamphetamine use and neonatal neurobehavioral outcome. Neurotoxicology and Teratology, 30 (1), 20-28. PMCID: 2358984.

Sowell, E. R., Jernigan, T. L., Mattson, S. N., Riley, E. P., Sobel, D. F., \& Jones, K. L. (1996). Abnormal development of the cerebellar vermis in children prenatally exposed to alcohol: size reduction in lobules I-V. Alcoholism, Clinical and Experimental Research, 20(1), 31-34.

Sowell, E. R., Thompson, P. M., Mattson, S. N., Tessner, K. D., Jernigan, T. L., Riley, E. P., et al. (2001a). Voxel-based morphometric analyses of the brain in children and adolescents prenatally exposed to alcohol. NeuroReport, 12(3), 515-523.

Sowell, E. R., Mattson, S. N., Thompson, P. M., Jernigan, T. L., Riley, E. P., \& Toga, A. W. (2001b). Mapping callosal morphology and cognitive correlates: effects of heavy prenatal alcohol exposure. Neurology, 57(2), 235-244.

Sowell, E. R., Thompson, P. M., Mattson, S. N., Tessner, K. D., Jernigan, T. L., Riley, E. P., et al. (2002a). Regional brain shape abnormalities persist into adolescence after heavy prenatal alcohol exposure. Cerebral Cortex, 12(8), 856-865.

Sowell, E. R., Thompson, P. M., Peterson, B. S., Mattson, S. N., Welcome, S. E., Henkenius, A. L., et al. (2002b). Mapping cortical gray matter asymmetry patterns in adolescents with heavy prenatal alcohol exposure. Neuroimage, 17(4), 1807-1819.

Sowell, E. R., Peterson, B. S., Thompson, P. M., Welcome, S. E., Henkenius, A. L., \& Toga, A. W. (2003). Mapping cortical change across the human life span. Nature Neuroscience, 6(3), 309-315.

Sowell, E. R., Thompson, P. M., \& Toga, A. W. (2004). Mapping changes in the human cortex throughout the span of life. The Neuroscientist, 10(4), 372-392.

Sowell, E. R., Lu, L. H., O'Hare, E. D., McCourt, S. T., Mattson, S. N., O'Connor, M. J., et al. (2007). Functional magnetic resonance imaging of verbal learning in children with heavy prenatal alcohol exposure. NeuroReport, 18(7), 635-639.

Sowell, E. R., Mattson, S. N., Kan, E., Thompson, P. M., Riley, E. P., \& Toga, A. W. (2008a). Abnormal cortical thickness and brainbehavior correlation patterns in individuals with heavy prenatal alcohol exposure. Cerebral Cortex, 18(1), 136-144. PMCID: 2770438.

Sowell, E. R., Johnson, A., Kan, E., Lu, L. H., Van Horn, J. D., Toga, A. W., et al. (2008b). Mapping white matter integrity and neurobehavioral correlates in children with fetal alcohol spectrum disorders. The Journal of Neuroscience, 28(6), 1313-1319.

Sowell, E. R., Leow, A. D., Bookheimer, S. Y., Smith, L. M., O'Connor, M. J., Kan, E., et al. (2010). Differentiating prenatal exposure to methamphetamine and alcohol versus alcohol and not methamphetamine using tensor-based brain morphometry and discriminant analysis. Journal of Neuroscience, 30(11), 38763885. PMCID: 2847574.
Spadoni, A. D., Bazinet, A. D., Fryer, S. L., Tapert, S. F., Mattson, S. N., \& Riley, E. P. (2009). BOLD response during spatial working memory in youth with heavy prenatal alcohol exposure. Alcohol Clin Exp Res, 33(12), 2067-2076. PMCID: 2789890.

Stanwood, G. D., \& Levitt, P. (2004). Drug exposure early in life: functional repercussions of changing neuropharmacology during sensitive periods of brain development. Current Opinion in Pharmacology, 4(1), 65-71.

Stanwood, G. D., Parlaman, J. P., \& Levitt, P. (2005). Anatomical abnormalities in dopaminoceptive regions of the cerebral cortex of dopamine D1 receptor mutant mice. The Journal of Comparative Neurology, 487(3), 270-282.

Stek, A. M., Fisher, B. K., Baker, R. S., Lang, U., Tseng, C. Y., \& Clark, K. E. (1993). Maternal and fetal cardiovascular responses to methamphetamine in the pregnant sheep. American Journal of Obstetrics and Gynecology, 169(4), 888-897.

Stek, A. M., Baker, R. S., Fisher, B. K., Lang, U., \& Clark, K. E. (1995). Fetal responses to maternal and fetal methamphetamine administration in sheep. American Journal of Obstetrics and Gynecology, 173(5), 1592-1598.

Sulzer, D., Sonders, M. S., Poulsen, N. W., \& Galli, A. (2005). Mechanisms of neurotransmitter release by amphetamines: a review. Progress in Neurobiology, 75(6), 406-433.

Swayze, V. W., 2nd, Johnson, V. P., Hanson, J. W., Piven, J., Sato, Y., Giedd, J. N., et al. (1997). Magnetic resonance imaging of brain anomalies in fetal alcohol syndrome. Pediatrics, 99(2), 232-240.

Ticku, M. K., Lowrimore, P., \& Lehoullier, P. (1986). Ethanol enhances GABA-induced 36Cl-influx in primary spinal cord cultured neurons. Brain Research Bulletin, 17(1), 123-126.

Wang, G. K. (1988). Cocaine-induced closures of single batrachotoxinactivated $\mathrm{Na}+$ channels in planar lipid bilayers. Journal of General Physiology, 92(6), 747-765. PMCID: 2228921.

Wang, H. Y., Runyan, S., Yadin, E., \& Friedman, E. (1995). Prenatal exposure to cocaine selectively reduces D1 dopamine receptormediated activation of striatal Gs proteins. The Journal of Pharmacology and Experimental Therapeutics, 273(1), 492-498.

Warner, T. D., Behnke, M., Eyler, F. D., Padgett, K., Leonard, C., Hou, W., et al. (2006). Diffusion tensor imaging of frontal white matter and executive functioning in cocaine-exposed children. Pediatrics, 118(5), 2014-2024.

Weissman, A. D., \& Caldecott-Hazard, S. (1995). Developmental neurotoxicity to methamphetamines. Clinical and Experimental Pharmacology \& Physiology, 22(5), 372-374.

Whitaker-Azmitia, P. M. (1998). Role of the neurotrophic properties of serotonin in the delay of brain maturation induced by cocaine. Annals of the New York Academy of Sciences, 846, 158-164.

Wilcoxon, J. S., Kuo, A. G., Disterhoft, J. F., \& Redei, E. E. (2005). Behavioral deficits associated with fetal alcohol exposure are reversed by prenatal thyroid hormone treatment: a role for maternal thyroid hormone deficiency in FAE. Molecular Psychiatry, 10(10), 961-971.

Woods, J. R., Jr. (1996). Adverse consequences of prenatal illicit drug exposure. Current Opinion in Obstetrics \& Gynecology, 8(6), 403-411.

Woods, J. R., Jr., Plessinger, M. A., \& Clark, K. E. (1987). Effect of cocaine on uterine blood flow and fetal oxygenation. JAMA, 257 (7), 957-961.

Wozniak, J. R., Mueller, B. A., Chang, P. N., Muetzel, R. L., Caros, L., \& Lim, K. O. (2006). Diffusion tensor imaging in children with fetal alcohol spectrum disorders. Alcohol Clin Exp Res, 30 (10), 1799-1806. PMCID: 2895767.

Zhang, X., Sliwowska, J. H., \& Weinberg, J. (2005). Prenatal alcohol exposure and fetal programming: effects on neuroendocrine and immune function. Experimental Biology and Medicine (Maywood), 230(6), 376-388. 